\begin{tabular}{ccc|} 
ISSN $=$ 1980-993X - doi:10.4136/1980-993X \\
www.ambi-agua.net \\
E-mail: ambi-agua@agro.unitau.br \\
Tel.: (12) 3625-4212
\end{tabular}

\title{
Importancia de la determinación de la humedad en estudios de infiltración y escorrentía superficial para períodos largos
}

\author{
(http://dx.doi.org/10.4136/ambi-agua.188) \\ Teresa Reyna, Santiago Reyna, María Lábaque, Fabián Fulginiti, César Riha, \\ Jorge Linares \\ ${ }^{1}$ Facultad de Ciencias Exactas, Físicas y Naturales. Universidad Nacional Córdoba. Av. Vélez Sarsfield 1611 \\ Córdoba, Córdoba, Argentina \\ e-mail: teresamaria.reyna@gmail.com; santiagoreyna@gmail.com; mlabaque@gmail.com; \\ fabianfulginiti@gmail.com; ingriha@gmail.com; linjorge@gmail.com
}

\section{RESUMEN}

La determinación de la humedad natural del suelo es primordial para resolver problemas vinculados a las necesidades de agua de riego, consideraciones ambientales y determinación de los excedentes hídricos. Para el cálculo del escurrimiento, se pueden adoptar modelos que consideran a la infiltración exclusivamente como una pérdida o modelos de cálculo de infiltración, que modelan el agua infiltrada. Los que utilizan el cálculo de la infiltración, consideran más adecuadamente la interacción de los procesos de infiltración - escorrentía y aportan información adicional sobre el fenómeno de infiltración que permite establecer las condiciones existentes de humedad en el suelo ante la ocurrencia de un nuevo evento (simulación para períodos largos). Estos modelos requieren resolver la ecuación de Richards y para ello es imprescindible determinar la relación entre la humedad del suelo - succión y conductividad hidráulica - succión que requieren de la determinación de las propiedades hidráulicas que pueden obtenerse mediante la medición del contenido de agua, perfiles de humedad. El objetivo de este trabajo fue la verificación de estas curvas de humedad en suelos loésicos del sur de la ciudad de Córdoba, Argentina. Para ello se realizaron mediciones que se contrastaron con las modelaciones de infiltración utilizando las funciones hidráulicas determinadas. Las mediciones se efectuaron utilizando tres sondas instaladas a distintas profundidades. Los resultados mostraron que los valores obtenidos con NETRAIN representan adecuadamente el comportamiento de humedecimiento y secado del suelo estudiado. La determinación de estas curvas servirá de base para los estudios futuros que incluyen el avance de agroquímicos en el suelo y su potencial capacidades de contaminar las aguas subterráneas tema fundamental para definir pautas de manejo ambiental.

Palabras claves: humedad en suelos; funciones hidráulicas; loess; zona vadosa; modelaciones hidrológicas.

\section{Importance of moisture determination in studies of infiltration and surface runoff for long periods}

\section{ABSTRACT}

The determination of the natural soil moisture is essential to solve problems related to irrigation water requirements, environmental considerations, and determination of surplus water. For the determination of runoff one can adopt models that consider exclusively the infiltration as a loss or one could use computational models of infiltration to model the infiltrated water. Models based on the infiltration calculation consider well the interaction 
REYNA, T.; REYNA, S.; LÁBAQUE, M.; FULGINITI, F.; RIHA, C.; LINARES, J. Facultad de Ciencias Exactas, Físicas y Naturales. Universidad Nacional Córdoba. Importancia de la determinación de la humedad en estudios de infiltración y escorrentía superficial para períodos largos. Ambi-Agua, Taubaté, v. 6, n. 2, p. 91-110, 2011. (doi:10.4136/ambi-agua.188)

between infiltration - runoff processes and provide additional information on the phenomenon of infiltration which establishes the existing conditions of moisture in the soil before the occurrence of a new event (simulation for long periods). These models require solving Richards's equation and for this purpose it is necessary to determine the relation between the soil moisture - suction and hydraulic conductivity - suction which require the determination of the hydraulic properties that can be obtained by measuring the water content by moisture profiles. The aim of this study was the verification of these moisture curves in loessic soils in the south of the city of Cordoba, Argentina. To do this, measurements were done and compared with results of infiltration models based on the determined hydraulic functions. The measurements were done using three probes installed at different depths. The results showed that the values obtained with NETRAIN adequately represent the behavior of wetting and drying conditions of the studied soil.The determination of these curves provided a basis for future studies that include the advancement of agricultural chemicals in the soil and its potential capacity to pollute groundwater, fundamental issue to define environmental management policies.

Keywords: moisture in soil; hydraulic functions; loess; vadose zone; hydrological modeling.

\section{Importância da determinação da umidade em estudos de infiltração e escoamento superficial por longos períodos}

\section{RESUMO}

A determinação da umidade natural do solo é essencial para resolver problemas relacionados com os requisitos da água de irrigação, as considerações ambientais e determinação de água em excesso. Para o cálculo do escoamento, podem ser adotados modelos que consideram a infiltração exclusivamente como uma perda ou modelos computacionais de infiltração, que modelam a água infiltrada. Aqueles que utilizam o cálculo de infiltração consideram melhor a interação dos processos de infiltração - enxurrada e fornecem informações adicionais sobre o fenômeno da infiltração, que permitem estabelecer as condições existentes de umidade no solo antes da ocorrência de um novo evento (simulação por longos períodos). Esses modelos requerem resolver a equação de Richards e para isso é essencial determinar a relação entre umidade do solo - sucção e condutividade hidráulica - sucção que exigem, por sua vez, a determinação das propriedades hidráulicas que podem ser obtidas pela medição de perfis de umidade do solo. O objetivo deste estudo foi a verificação dessas curvas de umidade em solos loésicos da cidade de Córdoba, Argentina. Para isso, realizaram-se medições que foram contrastadas com a modelagem da infiltração, usando-se as funções hidráulicas determinadas. As medições foram realizadas utilizando-se três sondas que foram instaladas em diferentes profundidades. Os resultados mostraram que os valores obtidos com NETRAIN representam adequadamente o comportamento de molhagem e secagem do solo estudado. A determinação dessas curvas servirá de base para estudos futuros que incluam o avanço de agrotóxicos no perfil do solo e sua capacidade potencial de poluição das águas subterrâneas, tema fundamental para definir diretrizes de gestão ambiental.

Palavras-chave: umidade do solo; funções hidráulicas; loess; zona vadosa; modelagens hidrológicas.

\section{INTRODUCCIÓN}

En la actualidad se ha tomado conciencia de la importancia de conocer y poder predecir el comportamiento hidrológico superficial, hidrogeológico e hidráulico de una manera más 
REYNA, T.; REYNA, S.; LÁBAQUE, M.; FULGINITI, F.; RIHA, C.; LINARES, J. Facultad de Ciencias Exactas, Físicas y Naturales. Universidad Nacional Córdoba. Importancia de la determinación de la humedad en estudios de infiltración y escorrentía superficial para períodos largos. Ambi-Agua, Taubaté, v. 6, n. 2, p. 91-110, 2011. (doi:10.4136/ambi-agua.188)

ajustada, como así también de las interrelaciones de estos sistemas. La parte de la precipitación que es considerada como pérdida por la hidrología clásica, es uno de los principales elementos en hidrogeología; es el factor que vincula los elementos de este complejo sistema.

Muchos investigadores tienen como objetivo resolver en forma conjunta los procesos de escorrentía e infiltración. En términos matemáticos, el escurrimiento en cursos superficiales y el flujo subterráneo en acuíferos freáticos están gobernados por ecuaciones diferenciales no lineales acopladas, definidas en áreas de geometría compleja, donde cada componente está caracterizada por una escala temporal muy disímil entre sí, aspecto que dificulta la solución conjunta de las ecuaciones (Paz et al., 2005).

Cuando se desea estudiar el escurrimiento de una cuenca utilizando modelos de infiltración, es común enfrentarse al problema de que existen pocos datos de las propiedades hidráulicas de los suelos. Sin embargo, en general, es posible contar con las curvas granulométricas y no resulta demasiado complicado realizar también otras mediciones geotécnicas simples con ensayos de suelo tradicionales: medición de la humedad residual y la humedad de saturación. Los parámetros determinados mediante mediciones son importantes y permiten obtener las funciones hidráulicas de los suelos de la cuenca.

Por otro lado, conociendo valores de las propiedades hidráulicas en forma discreta es posible definir funciones que establezcan relaciones continuas entre la humedad y la succión y la conductividad hidráulica y la succión. Aún cuando no es posible tener mediciones de las propiedades hidráulicas en todo el rango de succión, se pueden determinar las curvas de humedad-succión y conductividad-succión del suelo combinando los conocimientos de las propiedades de otros suelos y el conocimiento de otros parámetros del suelo en estudio (Reyna, 2000).

Breddin (1963) planteó una clasificación gráfica que permite establecer la conductividad hidráulica saturada a través de las curvas granulométricas de suelos. En particular, el conocimiento de la distribución granulométrica y los parámetros usuales obtenidos de los ensayos del suelo estándares permiten obtener las funciones de conductividad y humedad del suelo en forma aproximada.

El recurso suelo de Argentina ha sido la base principal del desarrollo económico del país. Largos períodos con grandes cosechas hicieron que al país se lo llamara "El granero del mundo". Desde otras latitudes, el nombre de Argentina se asociaba con amplias planicies de inagotables suelos profundos, oscuros, capaces de producir altos rendimientos de granos y carne de excelente calidad. Estos conceptos son relativamente válidos para la Pampa Húmeda que ocupa algo menos de un tercio del territorio, donde las planicies son dominantes, formadas por sedimentos modernos no consolidados, con pastizales naturales y clima templado (Hall et al., 1992). Sin embargo, los dos tercios restantes son altamente contrastantes, la mayor parte dominada por clima árido. Debe aplicarse riego para la producción de cultivos, la que sólo se practica en la proximidad de los ríos principales o en pequeños oasis de poca extensión en las áreas montañosas (Ibañez, 2010).

La provincia de Córdoba es la segunda provincia del país en nivel de actividad agropecuaria, por detrás de la Provincia de Buenos Aires. La agricultura de la provincia se caracteriza principalmente el cultivo de soja y maíz, seguido por el trigo, el sorgo y el girasol. Con respecto al maní, Córdoba concentra la práctica totalidad de la producción nacional en dicho rubro.

Con relación a estas actividades es importante destacar que las prácticas actuales de expansión de la actividad agrícola y la incorporación de agroquímicos ha llevado a la región a ser considerada como un sistema ambientalmente frágil, que brindan pocos servicios ambientales debido a la pérdida de biodiversidad y por encontrarse sometidos a distintos procesos de degradación encubiertos por una creciente dependencia de insumos. 
REYNA, T.; REYNA, S.; LÁBAQUE, M.; FULGINITI, F.; RIHA, C.; LINARES, J. Facultad de Ciencias Exactas, Físicas y Naturales. Universidad Nacional Córdoba. Importancia de la determinación de la humedad en estudios de infiltración y escorrentía superficial para períodos largos. Ambi-Agua, Taubaté, v. 6, n. 2, p. 91-110, 2011. (doi:10.4136/ambi-agua.188)

En función de lo expuesto es imprescindible avanzar en el conocimiento de los procesos de infiltración en estos suelos que son una fuente fundamental de ingresos para el país en este contexto se encaró la necesidad de la verificación de las curvas de humedad de los suelos loésicos al sur de la ciudad de Córdoba, república Argentina (Figura 1), determinadas teóricamente a través de estudios realizados en laboratorio, datos de otros suelos y curvas granulométricas y una importante aplicación posterior en modelaciones hidrológicas para períodos largos. La determinación de las curvas de humedad-succión y conductividad-succión de los loess servirán además para los estudios siguientes que incluyen el avance de los agroquímicos en los perfiles de los suelos y su potencial capacidades de contaminar las aguas subterráneas.
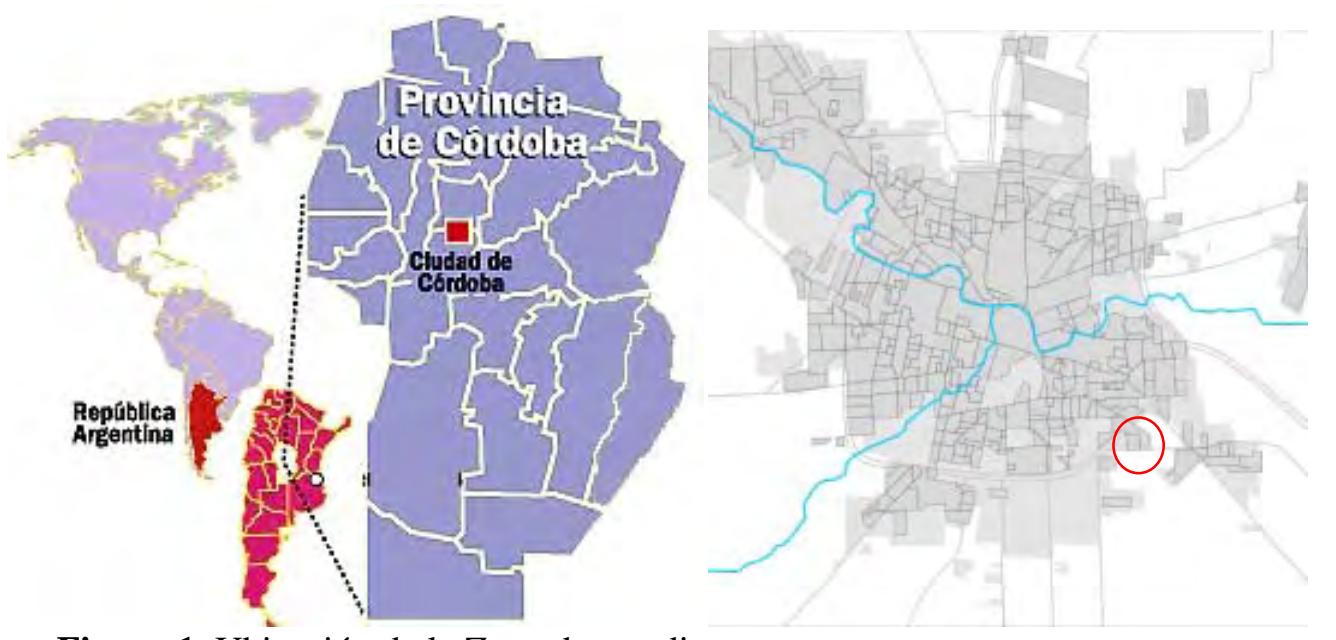

Figura 1. Ubicación de la Zona de estudio.

Con este fin se trabajó sobre una parcela experimental ubicada al sur de la Ciudad de Córdoba. En esta parcela se registraron de forma continua los eventos de precipitación y ausencia de esta ocurridas en un período de cuatro meses desde diciembre del año 2009. Se registraron también los valores de humedad experimentados por el suelo a distintas profundidades.

En este artículo se presenta las modelaciones de infiltración obtenidas utilizando el programa NETRAIN 3.0 (Reyna, 2008) realizados con datos probenientes de la parcela experimental considerando eventos de precipitación. Este programa fue desarrollado con el objetivo de generar un mecanismo que permita el cálculo de la infiltración por medio de la ecuación de Richards para su posterior ingreso a HEC-1. Desarrollándose esta aplicación de forma que sea posible su eventual incorporación al código fuente original de HEC-1.

NETRAIN 3.0 permite resolver la ecuación de Richards en diferencias finitas considerando las propiedades hidráulicas de los suelos de la base de datos de UNSODA (Leij et al., 1996). Al ser los datos de UNSODA una base de datos discreta, obtenida de las mediciones realizadas en distintas partes del mundo, NETRAIN interpola entre los datos originales para obtener una curva continua de conductividad - succión y humedad-succión. La salida del programa permite obtener el perfil de humedad para cada tiempo y la precipitación efectiva al descontar el agua que se infiltra en el suelo durante el proceso.

Los resultados obtenidos por este programa se contrastaron con mediciones realizadas sobre la parcela experimental. 
REYNA, T.; REYNA, S.; LÁBAQUE, M.; FULGINITI, F.; RIHA, C.; LINARES, J. Facultad de Ciencias Exactas, Físicas y Naturales. Universidad Nacional Córdoba. Importancia de la determinación de la humedad en estudios de infiltración y escorrentía superficial para períodos largos. Ambi-Agua, Taubaté, v. 6, n. 2, p. 91-110, 2011. (doi:10.4136/ambi-agua.188)

\subsection{Suelos Loésicos}

Unas de las principales dificultades que se encuentran para modelar los procesos de infiltración es conocer los parámetros de los suelos para lograr una adecuada representación de la realidad.

Los suelos más importantes desde el punto de vista agrícola están desarrollados en sedimentos eólicos cuaternarios que cubren las Planicies Chaco-Pampeanas, indicadas como ecoregiones Chaco Seco y Húmedo, Espinal y Pampa en la Figura 2 (Burkart et al., 1999). El material está formado por restos de rocas meteorizadas y también contiene cantidades significativas de vidrio volcánico, producto de la erupción de volcanes andinos.

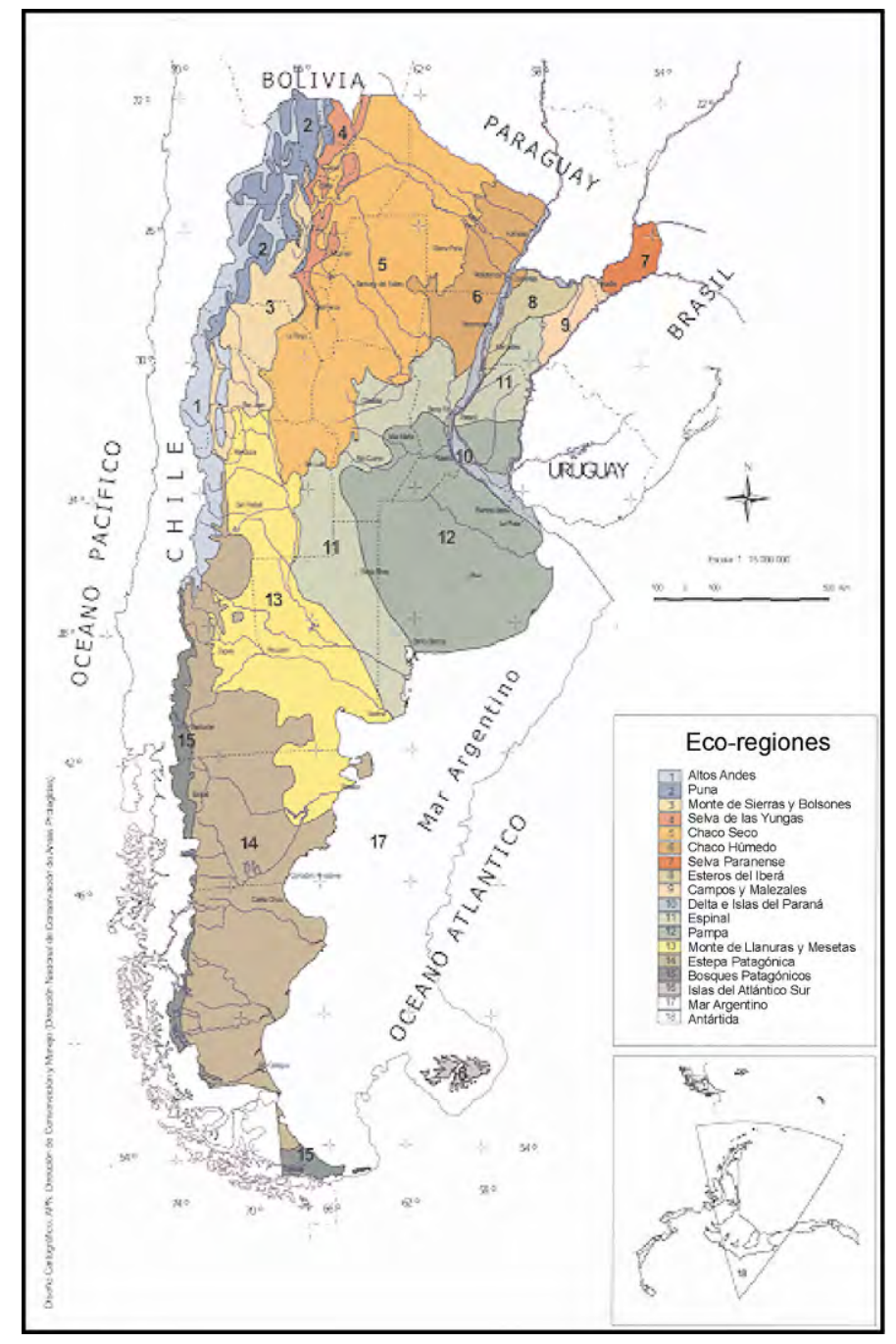

Figura 2. Eco-Regiones de Argentina. Modificado de: Burkart et al. (1999).

Este sedimento se conoce como Loess Pampeano debido a su similitud con materiales y depósitos loéssicos en otras partes del mundo (Frenguelli, 1955; Teruggi, 1957). Desde el punto de vista mineralógico el loess es rico en minerales meteorizables con cantidades conspicuas de calcio, potasio, fósforo y microelementos, así como materiales amorfos de origen volcánico (Scoppa, 1974).

En la Figura 3 (Pazos y Moscatelli, 1998) se muestra un ejemplo de la composición mineralógica promedio de suelos derivados de loess Pampeano, una fuente rica en nutrientes para las plantas. Las características físicas del loess Pampeano favorecen la formación de 
REYNA, T.; REYNA, S.; LÁBAQUE, M.; FULGINITI, F.; RIHA, C.; LINARES, J. Facultad de Ciencias Exactas, Físicas y Naturales. Universidad Nacional Córdoba. Importancia de la determinación de la humedad en estudios de infiltración y escorrentía superficial para períodos largos. Ambi-Agua, Taubaté, v. 6, n. 2, p. 91-110, 2011. (doi:10.4136/ambi-agua.188)

horizontes superficiales bien estructurados, profundos, oscuros y adecuados para el desarrollo de raíces.

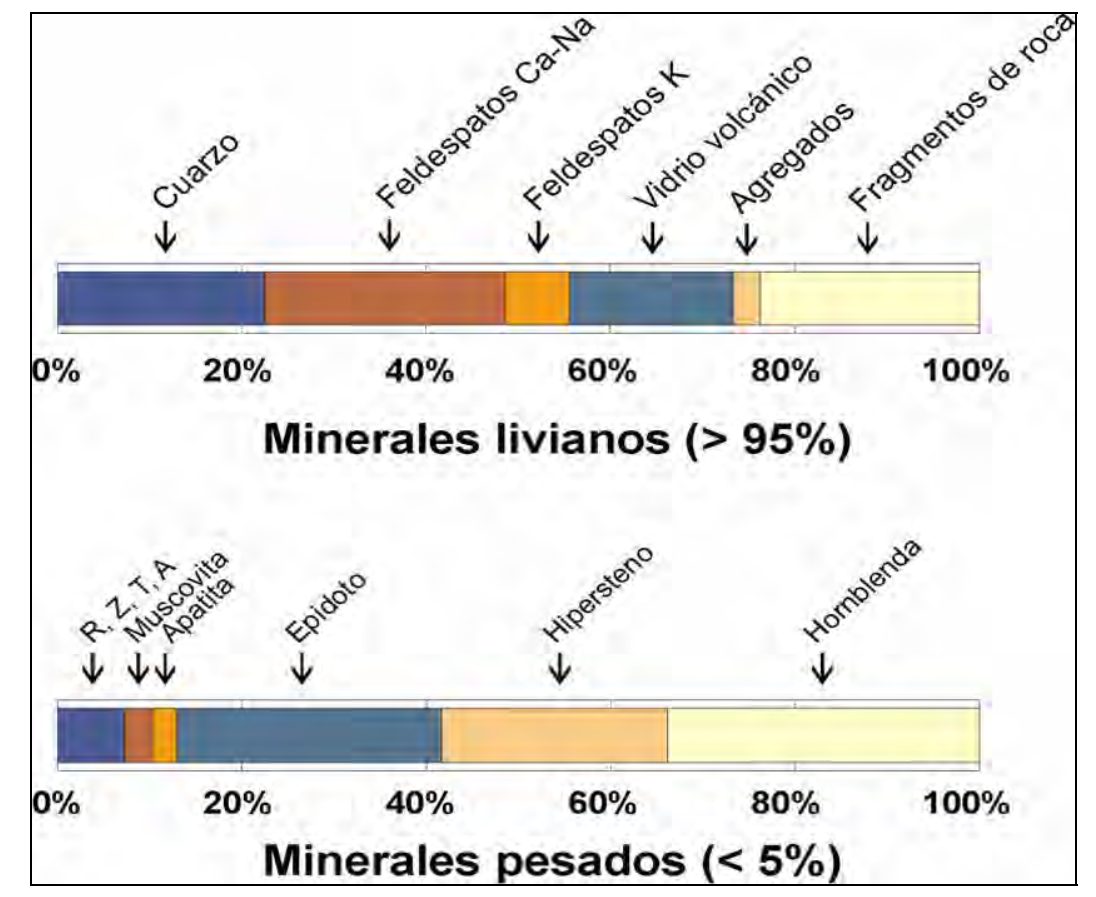

Figura 3. Composición mineralógica promedio de la fracción arena de seis perfiles de suelos del centro de la Provincia de Buenos Aires (Pazos y Moscatelli, 1998).

$\mathrm{R}$ = rutilo; $\mathrm{Z}$ = zircón; $\mathrm{T}$ = turmalina; $\mathrm{A}$ = anatasa

El loess de Córdoba en general está compuesto por: Arena fina $(>0,080 \mathrm{~mm}): 3,1 \%$, Limo (0,080 a 0,002 mm): 84,4\% y Arcilla $(<0,002 \mathrm{~mm}): 12,5 \%$. El Peso Unitario Seco promedio es $(\gamma \mathrm{d}): 13,14 \mathrm{kN} / \mathrm{m}^{3}$ y el grado de saturación varía entre el $27 \%$ y el $38 \%$ (Redolfi, 1993). En la Tabla 1 se presentan valores orientativos de los loess locales.

Tabla 1. Valores medios del loess de Córdoba, Argentina.

\begin{tabular}{l|c}
\hline PARÁMETRO & ENTORNO \\
\hline Humedad Natural Gravimétrica (w \%) & $\approx 20$ \\
Densidad Seca $\left(\mathrm{kN} / \mathrm{m}^{3}\right)$ & $12,5-15,3$ \\
Límite Líquido (\%) & $20-27$ \\
Índice Plástico (\%) & $2-6$ \\
Pasante Tamiz T $200(\%)$ & $85-99$ \\
\hline
\end{tabular}

\section{NETRAIN}

El modelo NETRAIN 3.0, resuelve la ecuación de Richards en diferencias finitas. En forma unidimensional la ecuación de Richards expresa:

$$
\frac{d \theta}{d t}=\frac{d}{d y}\left(K \frac{d H}{d y}+1\right)
$$

donde $\theta$ es la humedad volumétrica, $K$ la conductividad hidráulica y $H$ la succión. 
REYNA, T.; REYNA, S.; LÁBAQUE, M.; FULGINITI, F.; RIHA, C.; LINARES, J. Facultad de Ciencias Exactas, Físicas y Naturales. Universidad Nacional Córdoba. Importancia de la determinación de la humedad en estudios de infiltración y escorrentía superficial para períodos largos. Ambi-Agua, Taubaté, v. 6, n. 2, p. 91-110, 2011. (doi:10.4136/ambi-agua.188)

El modelo de flujo no saturado planteado para la fase líquida considera que la fase gaseosa se encuentra a presión atmosférica constante, es decir el modelo es monofásico.

La Ecuación 1 puede ser expresada en términos de diferencias finitas, obteniéndose la Ecuación 2.

$$
\begin{aligned}
& \theta_{(I, J)}=\theta_{(I-1, J)}+\frac{\left(K_{(I-1, J+1)}-K_{(I-1, J)}\right)}{D Y} * \frac{\left(H_{(I-1, J+1)}-H_{(I-1, J)}\right)}{D Y} * D T+ \\
& +K * \frac{\left(H_{(I-1, J+1)}-2 * H_{(I-1, J)}+H_{(I-1, J-1)}\right)}{D Y * D Y} * D T-\frac{\left(K_{(I-1, J+1)}-K_{(I-1, J)}\right)}{D Y} * D T
\end{aligned}
$$

donde el parámetro $\mathrm{K}$ se forma ponderando el valor de conductividad para las puntos contiguos en el paso de tiempo previo. Las variables que intervienen se pueden observar en la Figura 4.

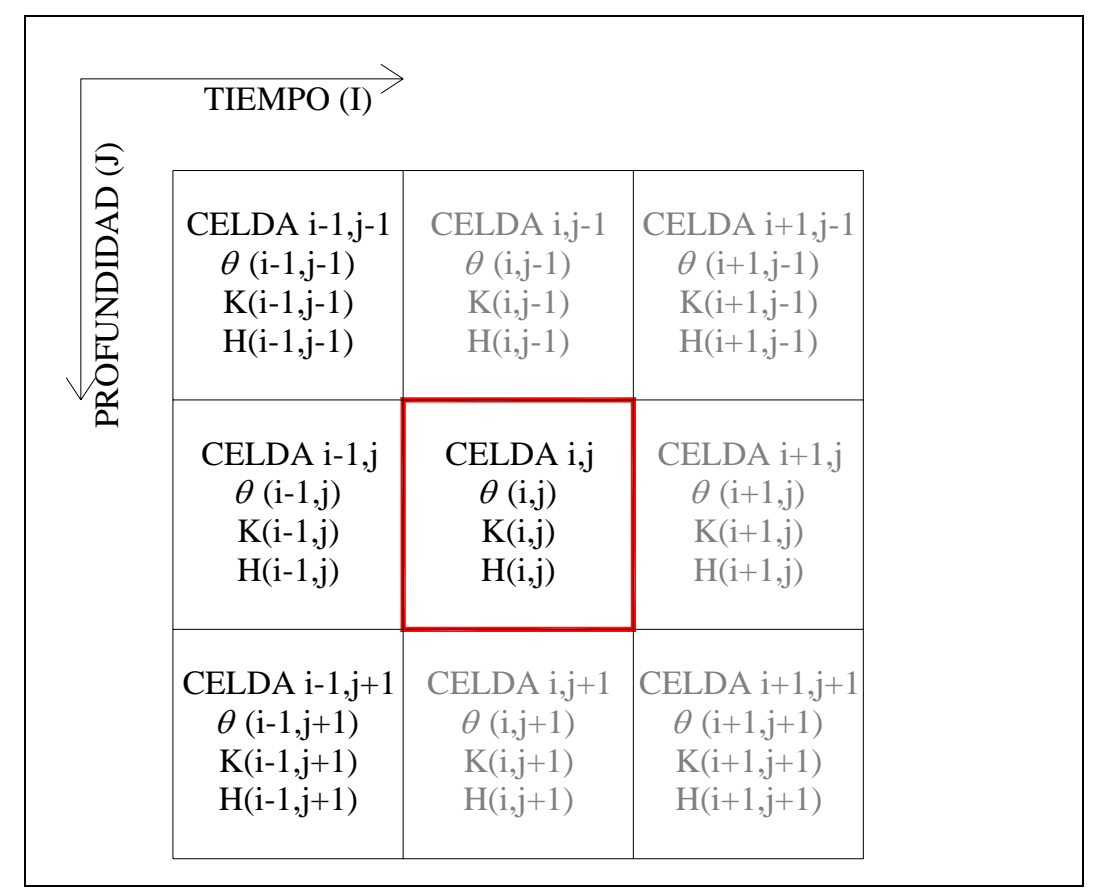

Figura 4. Esquema en diferencias finitas para la resolución del modelo unidimensional (Reyna, 2008).

El programa emplea un paso de tiempo fijo, de un minuto, para el cálculo computacional, el cual ha demostrado ser lo suficientemente pequeño para representar el proceso de escurrimiento en medios porosos. Dado el paso de tiempo, el programa fija el paso del elemento diferencial en el espacio cumpliendo con la condición de Courant para este tipo de problema.Asimismo, el programa determina la cantidad de elementos diferenciales y la cantidad de pasos de tiempo que abarcará la simulación para que el usuario pueda evaluar la aptitud de estos valores.

La modelación con NETRAIN se realizó para el período total desde donde se tomaron las mediciones para la misma se consideró una humedad superficial inicial de 0,21. Las condiciones precedentes de humedad son un patrón determinante en el comportamiento del suelo ante cualquier evento. Cuando se realizan simulaciones que no permiten manejar la evolución del secado y humedecimiento del suelo, la humedad antecedente cobra particular significación tanto en la determinación de las evoluciones de las variación de la humedad en el suelo como en la determinación de la escorrentía superficial asociada a dichos eventos 
REYNA, T.; REYNA, S.; LÁBAQUE, M.; FULGINITI, F.; RIHA, C.; LINARES, J. Facultad de Ciencias Exactas, Físicas y Naturales. Universidad Nacional Córdoba. Importancia de la determinación de la humedad en estudios de infiltración y escorrentía superficial para períodos largos. Ambi-Agua, Taubaté, v. 6, n. 2, p. 91-110, 2011. (doi:10.4136/ambi-agua.188)

donde una determinación precisa de la infiltración es fundamental para el cálculo correcto de la misma.

Las propiedades hidráulicas utilizadas en la modelación fueron determinadas con la metodología que se describe a continuación.

\subsection{Funciones Hidráulicas del Loess}

Para determinar las funciones hidráulicas de los mismos primero se procedió a comparar las propiedades hidráulicas de suelos del mundo con características físicas similares.

Los datos de los suelos y las mediciones se obtuvieron del programa UNSODA. La base de datos de UNSODA, proporciona las propiedades hidráulicas de los suelos no saturados (contenido de humedad, conductividad hidráulica y difusividad del agua en el suelo), propiedades básicas del suelo (distribución del tamaño de partículas, densidad, contenido de materia orgánica, etc.) e información adicional sobre el suelo y los procedimientos experimentales (Leij et al., 1996).

De acuerdo a los datos de suelos se realizaron las curvas de las propiedades hidráulicas medidas (ver Figura 5 y 6), en las mismas se puede observar que los valores son similares. Se pueden definir entonces envolventes que marcan el rango de variación de los valores de humedad y conductividad para este tipo de suelos y para distintas succiones.

El rango de variación de las propiedades hidráulicas de distintos tipo de suelos finos permite establecer un marco para las propiedades y para los estudios de infiltración.

La curva correspondiente a la envolvente de las conductividades hidráulicas superior corresponde a un suelo hipotético con función de conductividad hidráulica - succión por encima de las conductividades de los suelos analizados, lo mismo se realizó para la función hidráulica límite inferior de las conductividades hidráulicas y de las curvas de humedadsucción.

Estas funciones hidráulicas se determinaron utilizando el modelo de van GenuchtenMualem.

Van Genuchten (1980) presentó una ecuación para el cálculo del grado de saturación efectiva, la cual tiene ventajas para su implementación en los modelos de cálculo de flujo en medios porosos no saturados,

$$
S_{e}=\frac{1}{\left[1+(\alpha h)^{n}\right]^{m}}
$$

donde $\alpha, \mathrm{n}$ y m son constantes empíricas. La ecuación tiene como límite la expresión de Brooks y Corey con $\lambda=\mathrm{mn}$. Cuando $\mathrm{n}$ tiende a infinito (mientras el producto mn es constante e igual a 0,4), aparece la curva de Brooks y Corey, con un determinado valor de entrada de aire. Las restricciones usuales utilizadas para la ecuación de Van Genuchten son m = 1-1/n y $\mathrm{m}=1-2 / \mathrm{n}$. Los resultados más estables se obtienen generalmente cuando se utilizan las restricciones para una serie incompleta de datos.

El modelo de Mualem (1976) expresa a la conductividad hidráulica en función del grado de saturación:

$$
K\left(S_{e}\right)=K_{s} S_{e}^{l}\left[\frac{f\left(S_{e}\right)}{f(1)}\right]^{2}
$$

donde

$$
f\left(S_{e}\right)=\int_{0}^{S_{e}} \frac{1}{h(x)} d x
$$

$K_{s}$ es la conductividad hidráulica en estado de saturación y $l$ es un parámetro de la conectividad de poros estimado por Mualem (1976) que en general vale 0,5. 
REYNA, T.; REYNA, S.; LÁBAQUE, M.; FULGINITI, F.; RIHA, C.; LINARES, J. Facultad de Ciencias Exactas, Físicas y Naturales. Universidad Nacional Córdoba. Importancia de la determinación de la humedad en estudios de infiltración y escorrentía superficial para períodos largos. Ambi-Agua, Taubaté, v. 6, n. 2, p. 91-110, 2011. (doi:10.4136/ambi-agua.188)

Cuando $n$ es menor que 1 no se puede predecir la función de conductividad, esta característica es una limitación importante del caso de variables $m$ y $n$. Van Genuchten, Leij y Yates recomiendan el uso de las variables $m, n$ sólo para el caso de tener datos bien definidos de humedad, y el uso de la restricción $m=1-1 / n$ para todos los otros casos.

Las ecuaciones para la conductividad y la difusividad $(D=K d h / d \theta)$ asumen que el valor de $K_{s}$ está bien definido y puede ser medido fácilmente, esto es cierto para suelos granulares, pero para los suelos no alterados esto no es cierto. La inspección de las curvas de conductividad y difusividad muestra que un pequeño cambio en el contenido de humedad produce cambios de varios órdenes en $K$ y $D$, lo que indica que pequeños errores en la medición del contenido de humedad cerca de la saturación pueden producir grandes errores en la estimación de la conductividad hidráulica saturada del suelo. Las consideraciones teóricas y experimentales sugieren que $K_{s}$ no debe utilizarse para ajustar los modelos de conductividad hidráulica (Jackson, et al., 1965; Green y Corey, 1971). Si se propone algún punto arbitrario de la conductividad hidráulica $\left(K_{0}\right)$ asociado a algún valor de humedad $\left(\theta_{0}\right)$ el modelo de Mualem puede ser redefinido como:

$$
K\left(S_{e}\right)=K\left(S_{e_{0}}\right)\left[\frac{S_{e}}{S_{e_{0}}}\right]^{l}\left[\frac{f\left(S_{e}\right)}{f\left(S_{e_{0}}\right)}\right]^{2}
$$

donde el grado de saturación es:

$$
S_{e_{0}}=S_{e}\left(\theta_{0}\right)=\frac{\theta_{0}-\theta_{r}}{\theta_{s}-\theta_{r}}
$$

Los parámetros del suelo hipotético correspondiente a la función hidráulica conductividad superior son humedad de saturación $0,467 \mathrm{~cm}^{3} / \mathrm{cm}^{3}$ y conductividad saturada $2,92 \mathrm{~cm} / \mathrm{h}$; para el suelo hipotético límite conductividad inferior: humedad de saturación $0,30 \mathrm{~cm}^{3} / \mathrm{cm}^{3}$ y conductividad hidráulica saturada $0,004 \mathrm{~cm} / \mathrm{h}$.

Para los suelos hipotéticos límites de la humedad se obtuvieron los siguientes parámetros: superior, humedad de saturación $0,47 \mathrm{~cm}^{3} / \mathrm{cm}^{3}$ y conductividad saturada 2,91 $\mathrm{cm} / \mathrm{h}$ y; para el suelo hipotético límite humedad inferior: humedad de saturación $0,30 \mathrm{~cm}^{3} / \mathrm{cm}^{3}$ y conductividad hidráulica saturada 0,006 cm/h (Reyna, 2000) (Figura 5 y Figura 6).

La determinación de estos parámetros es particularmente significativa en los suelos loésicos, en los que el agua tiene un rol muy importante en la formación y comportamiento posterior de los mismos (Rinaldi, 1994).

De los análisis realizados de las curvas de propiedades hidráulicas de otros suelos finos y de la comparación con los datos de campo y laboratorio sobre el loess pampeano, particularmente el de la zona sur de Córdoba, se estableció que el mismo tiene las siguientes características hidráulicas medias: humedad residual $0,10 \mathrm{~cm}^{3} / \mathrm{cm}^{3}$; humedad de saturación $0,36 \mathrm{~cm}^{3} / \mathrm{cm}^{3}$; conductividad saturada $61 \mathrm{~cm} /$ día. 
REYNA, T.; REYNA, S.; LÁBAQUE, M.; FULGINITI, F.; RIHA, C.; LINARES, J. Facultad de Ciencias Exactas, Físicas y Naturales. Universidad Nacional Córdoba. Importancia de la determinación de la humedad en estudios de infiltración y escorrentía superficial para períodos largos. Ambi-Agua, Taubaté, v. 6, n. 2, p. 91-110, 2011. (doi:10.4136/ambi-agua.188)

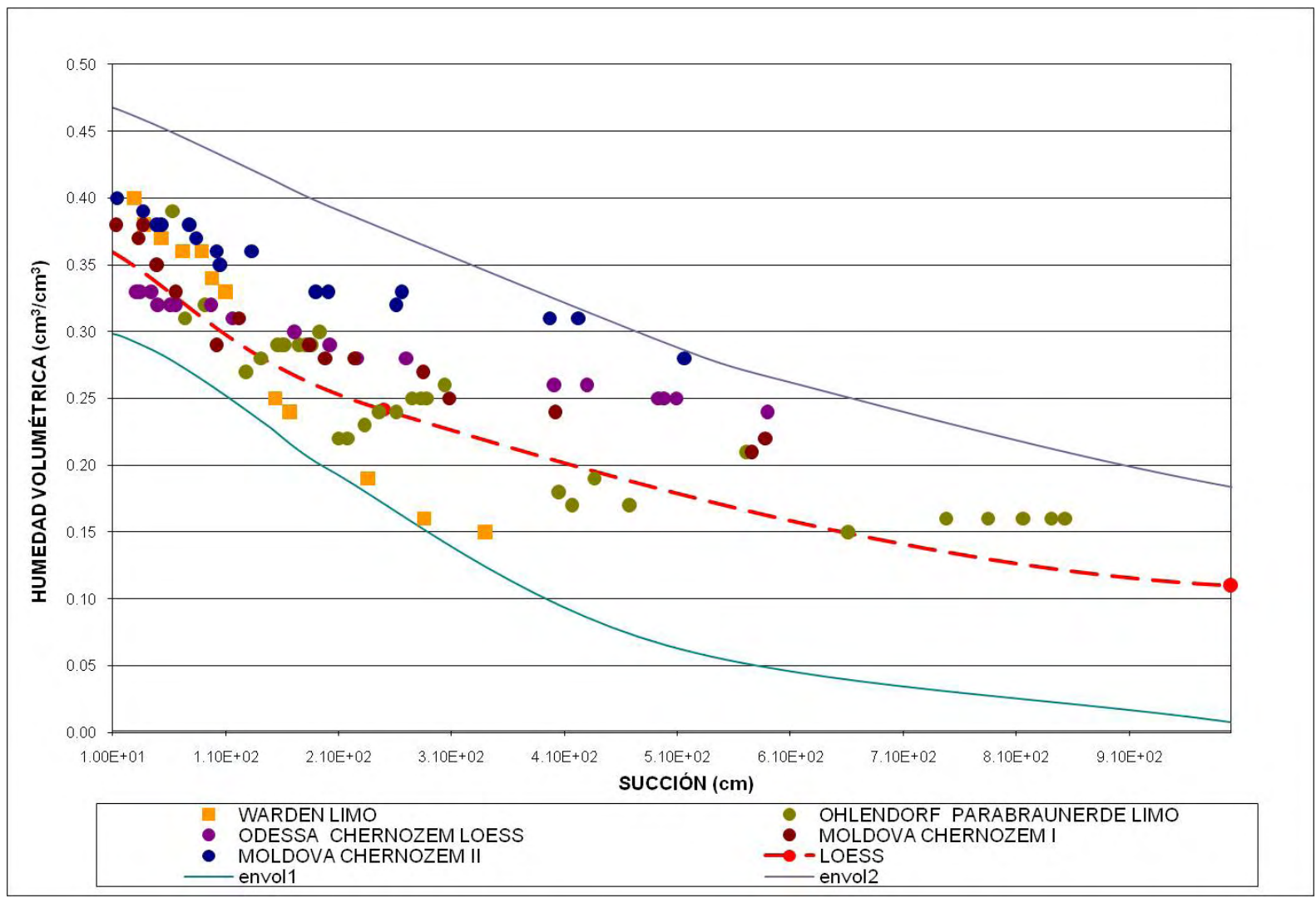

Figura 5. Curvas de humedad - succión, suelos; Warden Limo (Rockhold et al., 1988), Ohlendorf (Plagge et al., 1990), Odessa (Shein, 1990), Moldova Chernozem I y II (Meschtankova, 1989).

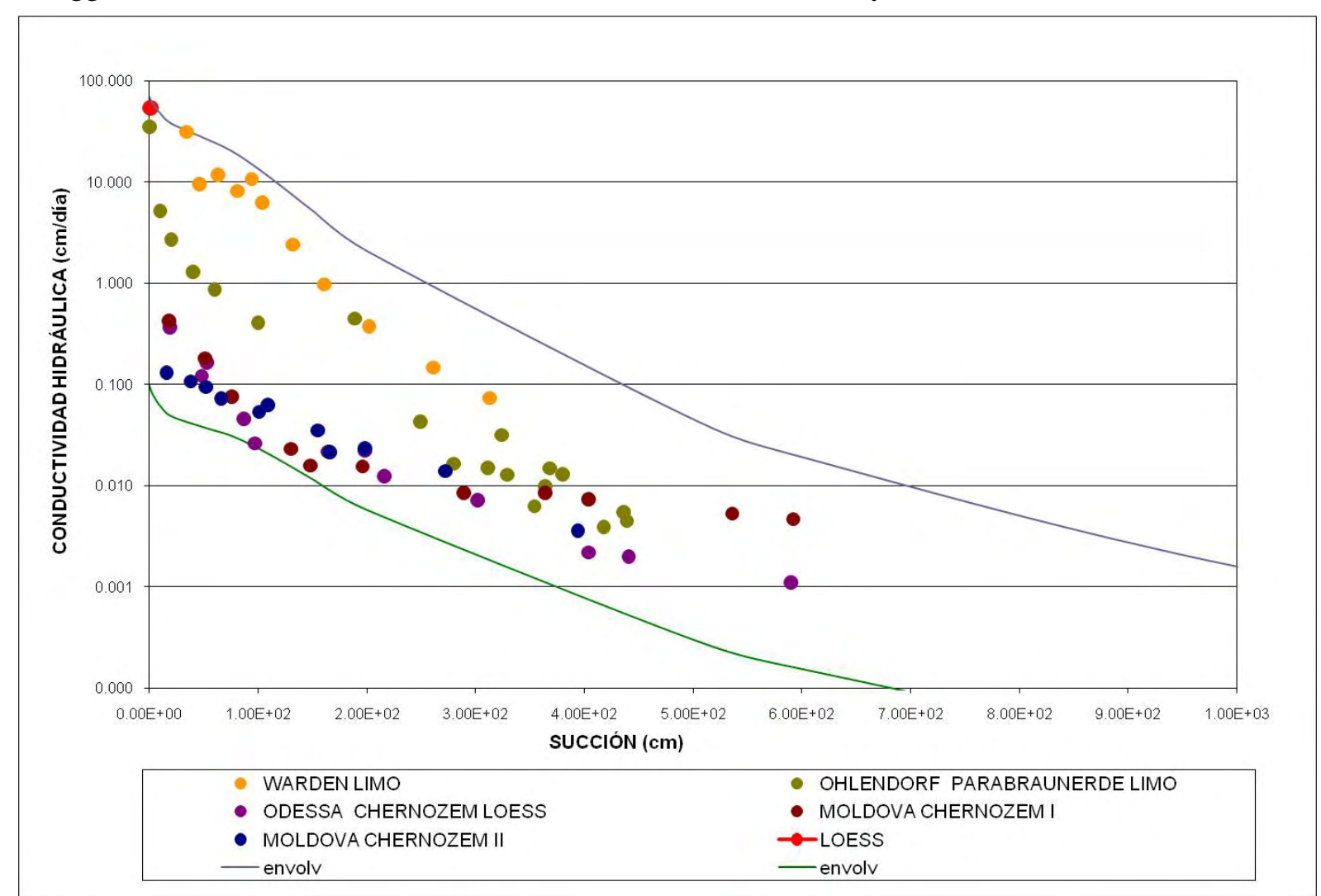

Figura 6. Curvas de Conductividad - succión, suelos; Warden Limo (Rockhold et al., 1988), Ohlendorf (Plagge et al., 1990), Odessa (Shein, 1990), Moldova Chernozem I y II (Meschtankova, 1989). 
REYNA, T.; REYNA, S.; LÁBAQUE, M.; FULGINITI, F.; RIHA, C.; LINARES, J. Facultad de Ciencias Exactas, Físicas y Naturales. Universidad Nacional Córdoba. Importancia de la determinación de la humedad en estudios de infiltración y escorrentía superficial para períodos largos. Ambi-Agua, Taubaté, v. 6, n. 2, p. 91-110, 2011. (doi:10.4136/ambi-agua.188)

\subsection{Mediciones de Campo}

Con el objetivo de verificar las modelaciones obtenidas con el modelo NETRAIN; se hacía necesario contrastar los resultados de humedecimiento y secado con mediciones de campo.

Existen distintos métodos para estimar la humedad de suelo en campo (muestreo gravimétrico, sonda de neutrones, tensiómetros, psicrómetros, etc.). Desafortunadamente, cada uno de estos métodos tiene dificultades que los alejan de lo ideal (Vita Serman et al., 2006).

Los métodos dieléctricos (incluyendo sensores de capacitancia (FDR), alta frecuencia, radio frecuencia, microondas y TDR) proveen una buena precisión a pesar de ser de medición indirecta si, como todo método indirecto de medición de humedad de suelo, se realiza una calibración en el lugar específico de utilización para definir la relación entre el contenido volumétrico del suelo y su coeficiente dieléctrico.

En este trabajo el método elegido para las mediciones de humedad en el campo fue el de sensores de capacitancia los que ofrecen una excelente alternativa al TDR, por su bajo coste y bajo consumo energético (Bogena et al., 2007).

Las mediciones se realizaron por el período de cuatro meses a partir de diciembre de 2009 en una parcela experimental de $5 \mathrm{~m}$ x $5 \mathrm{~m}$ con escasa pendiente hacia el centro donde un receptáculo conectado a un ducto enterrado conduce los excedentes hacia el exterior del predio, por medio del cual se aforaban los caudales excedentes. La cubierta vegetal es césped gramillón, el cual es cortado quincenalmente para mantener su altura entre 1,5 y 4 cm.

Con el fin de realizar un seguimiento de la humedad del suelo se instalaron sensores de humedad a tres profundidades distintas.

Se registraron la respuesta de los sensores ante la ocurrencia de eventos de precipitación. El intervalo de lectura en cada sensor fue seteado en 5 minutos.

\section{MATERIALES Y MÉTODOS}

Los sensores utilizados para medir la humedad del suelo fueron sensores de capacitancia adquiridos a la empresa DECAGON y corresponden a los modelos (Figura 7):

- 10 - HS (Denominado a partir de este momento P1): Colocado a $10 \mathrm{~cm}$ bajo el nivel del terreno

- 5 - TE (P2): Colocado a $30 \mathrm{~cm}$ bajo el nivel del terreno

- 5 - EC (P3): Colocado a $80 \mathrm{~cm}$ bajo el nivel del terreno.
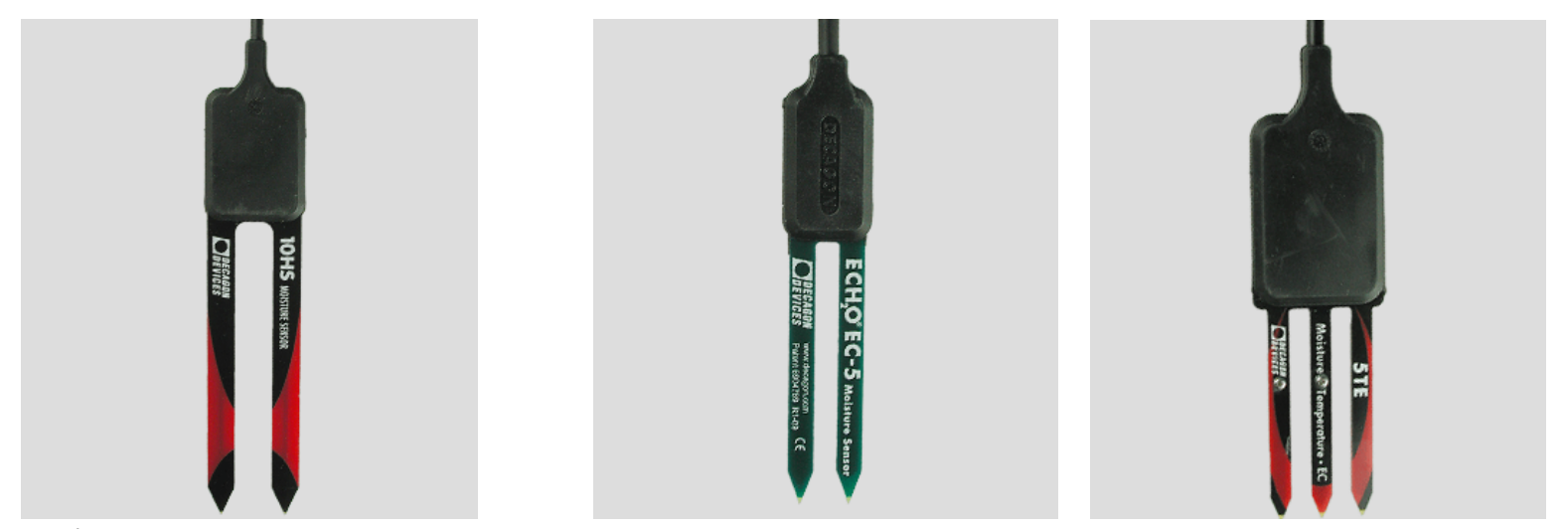

Figura 7. Imágenes de los sensores 10-HS, 5 - EC y 5 -TE.

Los eventos de precipitación se midieron con un pluviógrafo de una sola cuchara autodescargable marca Decagon Modelo ECHO - RAIN de exactitud +/- 2\%. 
REYNA, T.; REYNA, S.; LÁBAQUE, M.; FULGINITI, F.; RIHA, C.; LINARES, J. Facultad de Ciencias Exactas, Físicas y Naturales. Universidad Nacional Córdoba. Importancia de la determinación de la humedad en estudios de infiltración y escorrentía superficial para períodos largos. Ambi-Agua, Taubaté, v. 6, n. 2, p. 91-110, 2011. (doi:10.4136/ambi-agua.188)

Los datos fueron recogidos mediante un datalogger modelo EM-50 el cual tiene 5 canales de entrada, resolución A/D de 32 bits y excitación de $3 \mathrm{~V}$ por canal. Posteriormente los datos recogidos fueron procesados identificando y eliminando errores de muestreo.

\subsection{Calibración}

Las sondas Decagon de ECH2O miden el contenido volumétrico de agua del suelo mediante la medición de la constante dieléctrica del suelo, que es función del contenido de agua. Sin embargo, no todos los suelos tienen las mismos propiedades eléctricas. Debido a las variaciones en la textura del suelo y la salinidad, la precisión en los resultados obtenidos utilizando la calibración genérica para Sondas ECH2O es de aproximadamente $\pm 3.4 \%$ para la mayoría de suelos minerales de textura mediana a fina y una exactitud para los suelos de alta salinidad y de textura gruesa que puede variar hasta $\pm 10 \%$. Sin embargo, la precisión aumenta $\pm 2.1 \%$ para todos los suelos con la calibración del suelo específico.

Decagon recomienda que los usuarios de sondas ECH2O realicen una calibración del suelo específica para la mejor precisión posible en las mediciones del contenido volumétrico de agua. (Duglas, 2009), por lo que se procedió a realizar la calibración para el tipo de suelo a estudiar.

Se obtuvieron las curvas de calibración específicas tomando tres muestras de 7,5 x 7,2 $\mathrm{cm}$, empaquetadas en las que se insertaron cada sensor.

Partiendo de saturación, las muestras se van secando progresivamente al aire y se van tomando medidas simultáneas con el sensor y del peso de la muestra con la balanza de laboratorio. Finalmente se determina el peso seco tras secado en estufa a $105^{\circ} \mathrm{C}$. A partir del los pesos obtenidos y con el peso seco se calcula $\theta$ como cociente entre la diferencia de pesadas entre ambos pesos y el volumen de la muestra.

Para dicho ajuste se procedió a correlacionar las lecturas que arroja el sensor (RAW) y la humedad del suelo medido en el laboratorio para cada sensor. RAW es la unidad de almacenamiento del datalogger. Las curvas de calibración y las ecuaciones de calibración obtenidas se observan en las Figuras 8 y 9.

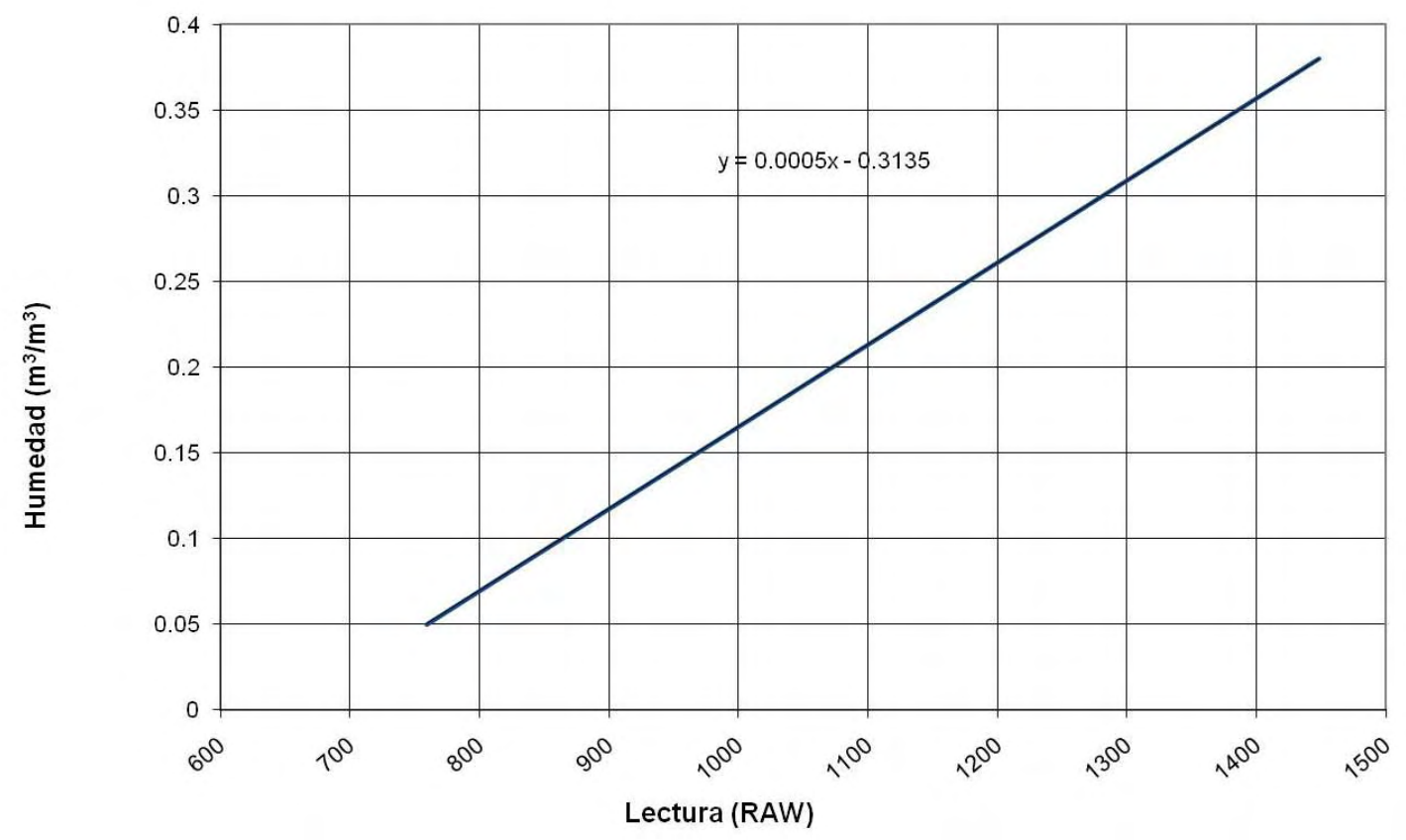

Figura 8. Funciones de correlación lectura (RAW) vs humedad $\left(\mathrm{m}^{3} / \mathrm{m}^{3}\right)$ para el sensor 10-HS. 
REYNA, T.; REYNA, S.; LÁBAQUE, M.; FULGINITI, F.; RIHA, C.; LINARES, J. Facultad de Ciencias Exactas, Físicas y Naturales. Universidad Nacional Córdoba. Importancia de la determinación de la humedad en estudios de infiltración y escorrentía superficial para períodos largos. Ambi-Agua, Taubaté, v. 6, n. 2, p. 91-110, 2011. (doi:10.4136/ambi-agua.188)

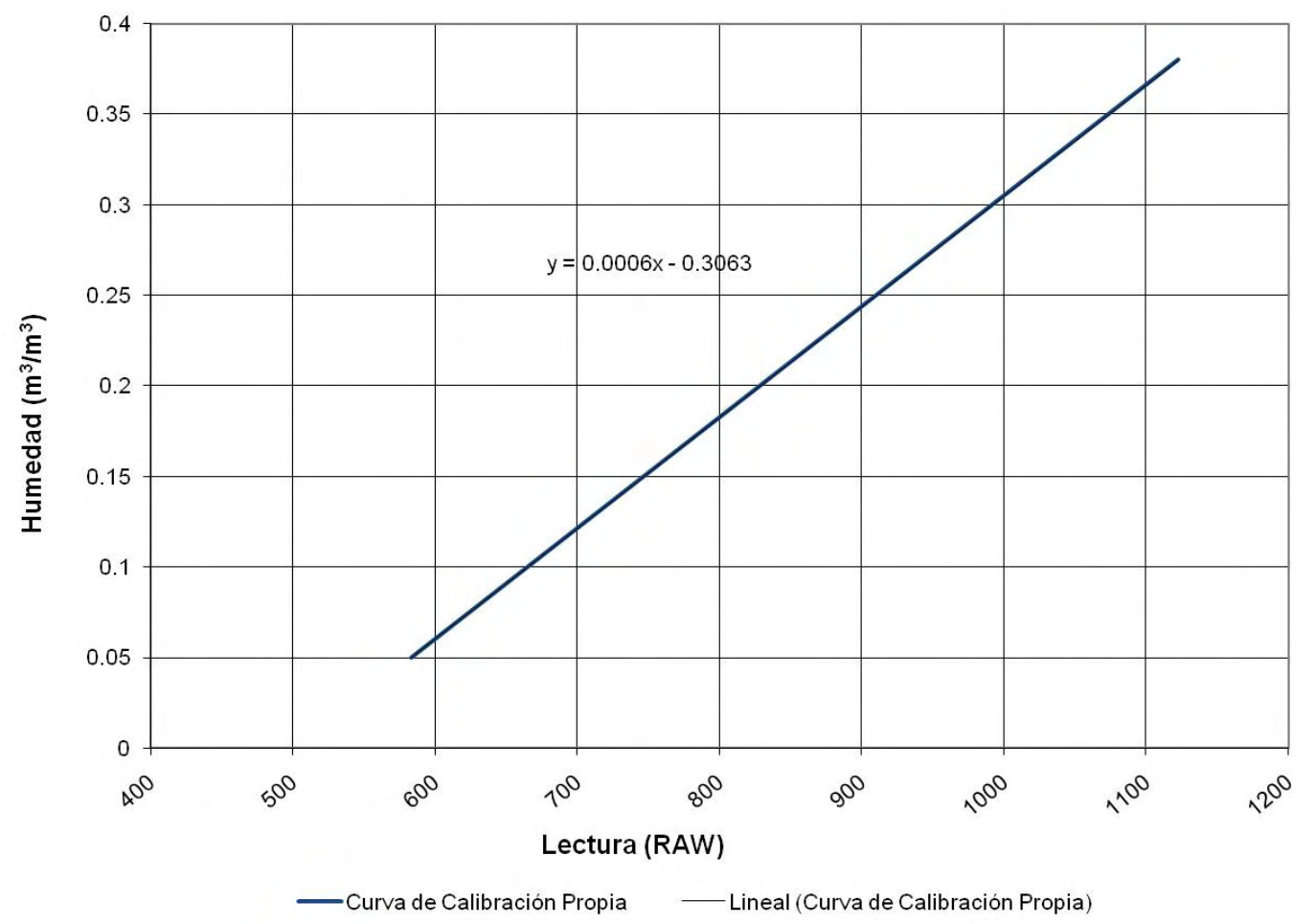

Figura 9. Función de correlación lectura (RAW) vs humedad $\left(\mathrm{m}^{3} / \mathrm{m}^{3}\right)$ para el sensor 5-EC.

Una vez calibrado los sensores para el suelo estudiado, se procedió a graficar las curvas de humedecimiento - secado en función del tiempo para los distintos sensores.

Las curvas de humedecimiento - secado medidas en función del tiempo para los sensores se presentan en las en las Figuras 10, 11 y 12. En las Figuras 10 y 11 se observa la variación que experimenta la humedad frente a los eventos de precipitación desde un valor de 0,37 que corresponde a la saturación de la superficie.

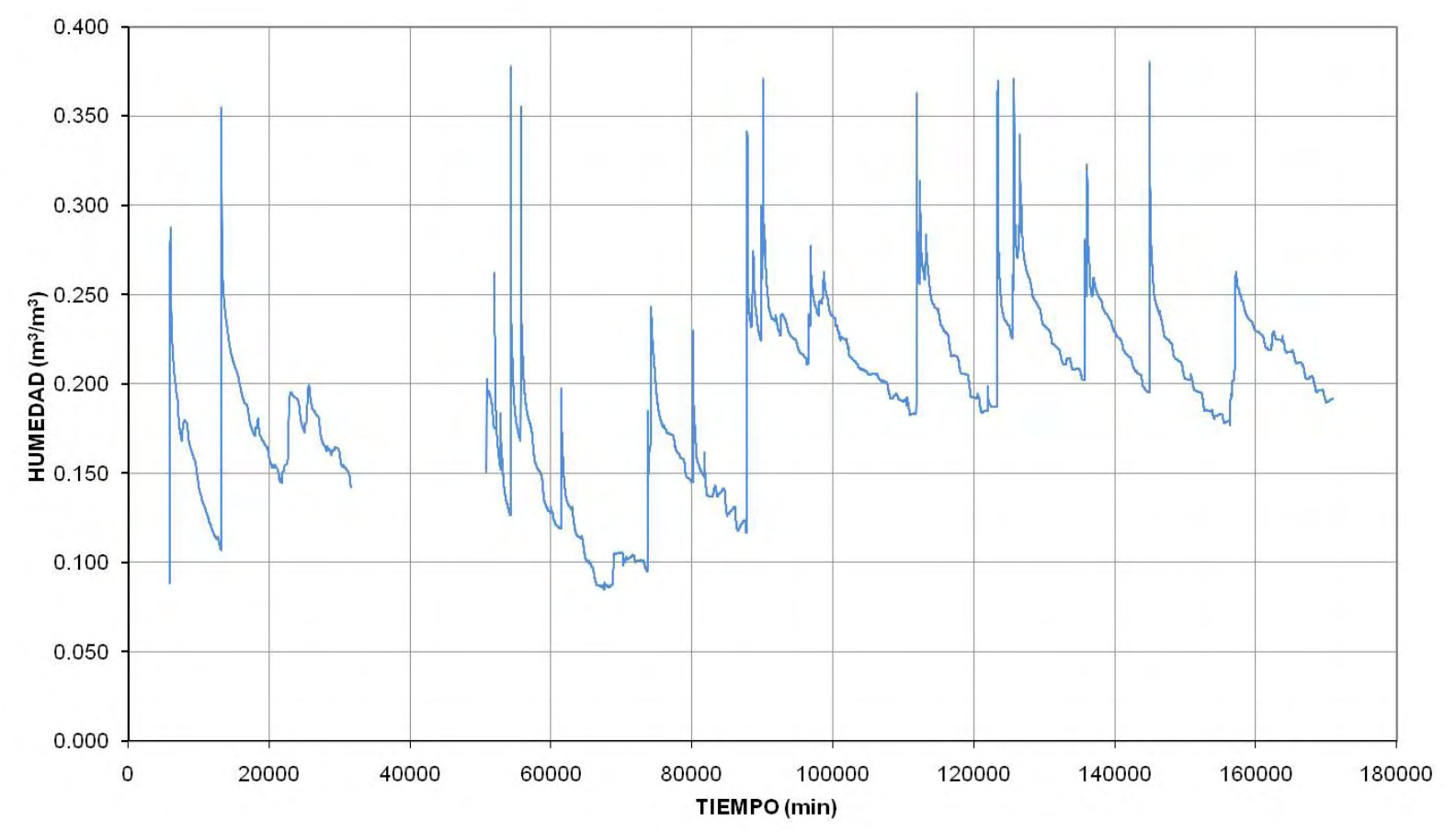

Figura 10. Curva de Humedecimiento - Secado - Sensor P1: $h=10 \mathrm{~cm}$. 
REYNA, T.; REYNA, S.; LÁBAQUE, M.; FULGINITI, F.; RIHA, C.; LINARES, J. Facultad de Ciencias Exactas, Físicas y Naturales. Universidad Nacional Córdoba. Importancia de la determinación de la humedad en estudios de infiltración y escorrentía superficial para períodos largos. Ambi-Agua, Taubaté, v. 6, n. 2, p. 91-110, 2011. (doi:10.4136/ambi-agua.188)

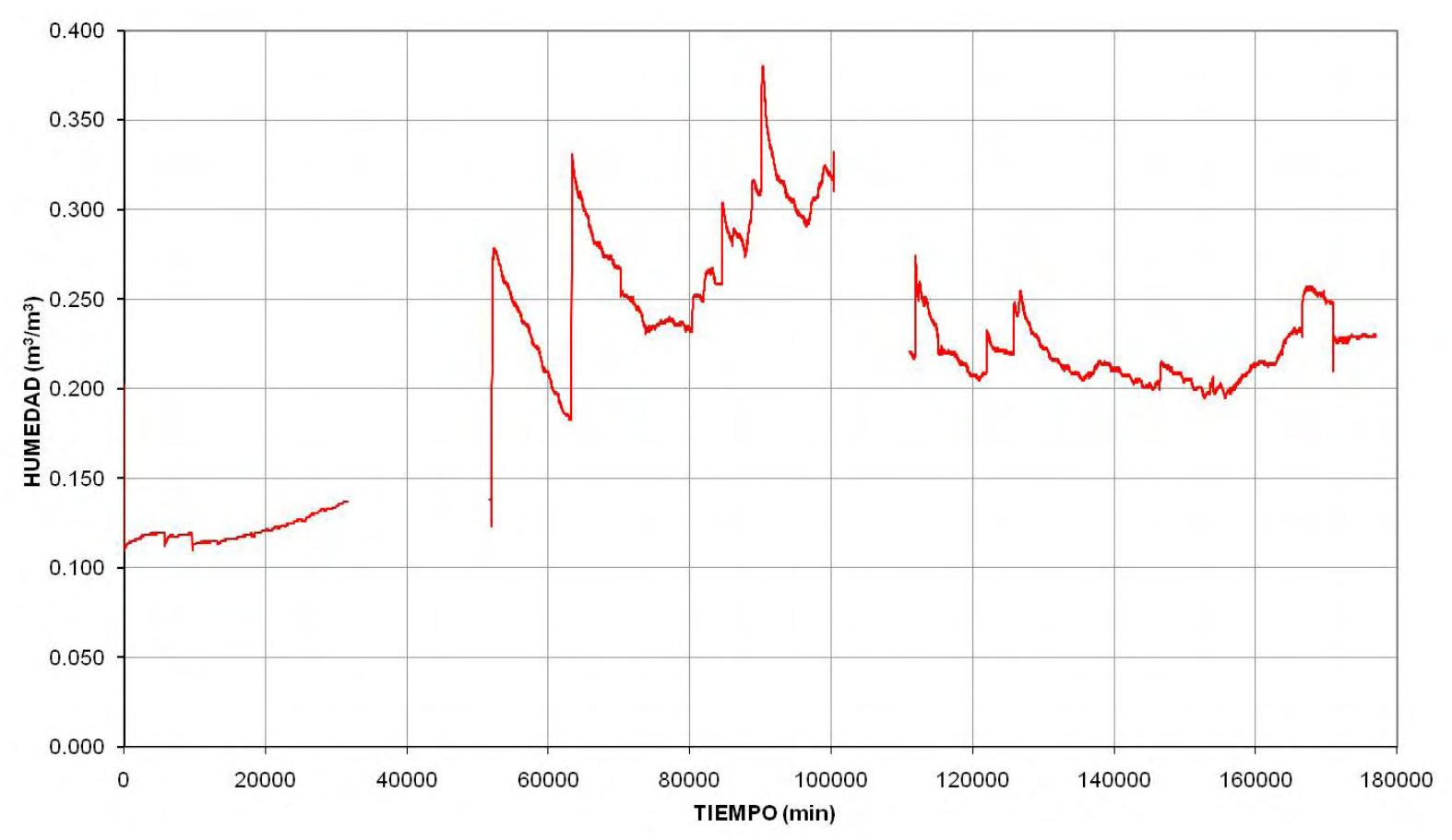

Figura 11. Curva de Humedecimiento - Secado - Sensor P2: $h=30 \mathrm{~cm}$.

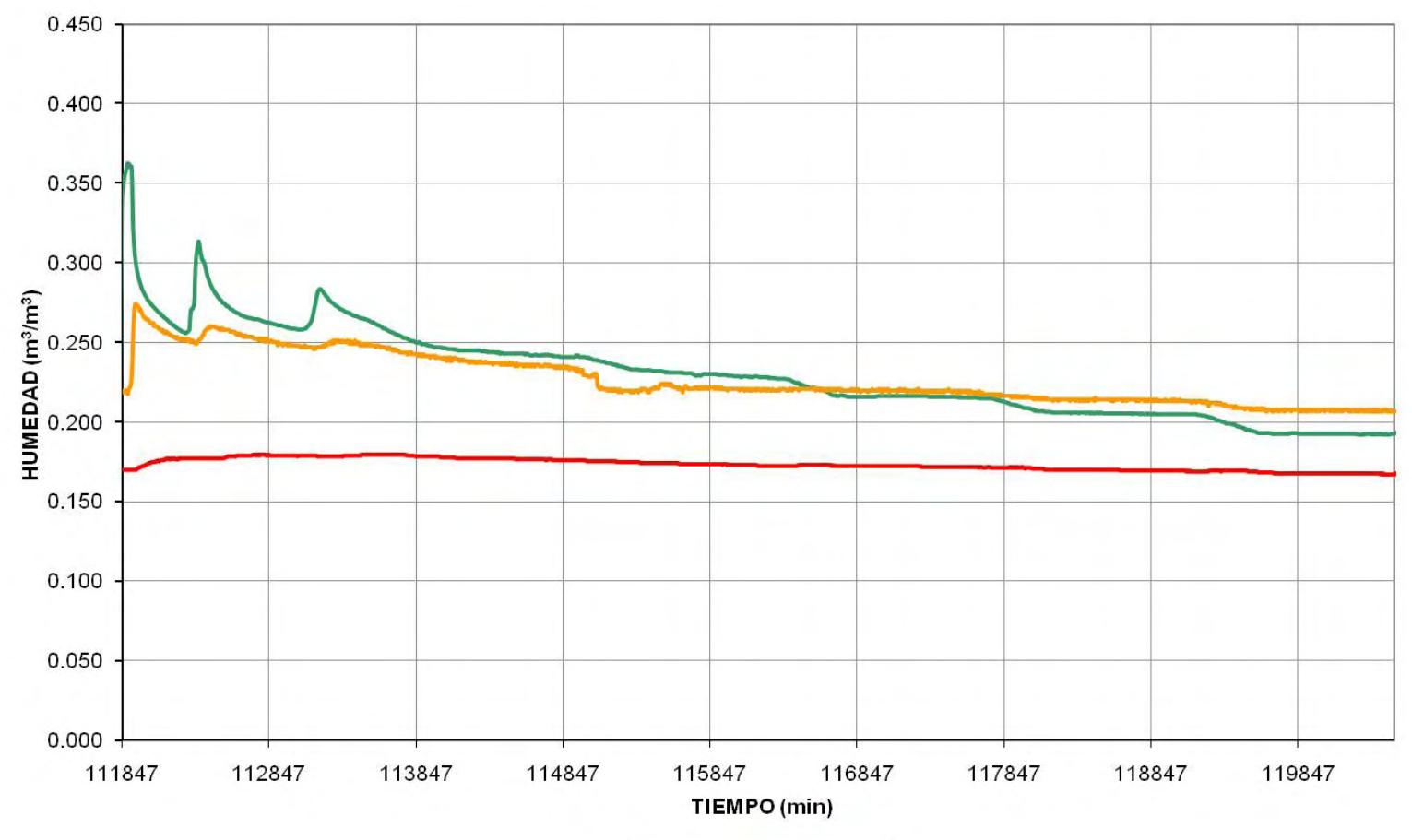

$-\mathrm{P} 1-\mathrm{P} 2-\mathrm{P} 3$

Figura 12. Curva de Humedecimiento -Secado. Sensores P1 $(10 \mathrm{~cm}), \mathrm{P} 2(30 \mathrm{~cm})$ y P3 $(80 \mathrm{~cm})$.

La calibración anterior se verificó con suelo de otra parcela ubicada más al sur lote del instituto del INTA en Córdoba coordenadas geográficas 63 44'44.91', Oeste 31 52' 19.08', Sur, en esta nueva parcela se colocaron los sensores luego de realizada la calibración. Al igual que para el primer caso se tomaron muestras del suelo y se las ensayaron en laboratorio. Los resultados de las calibraciones para el suelo de la nueva parcela se presentan en las Figuras 13 y 14. 
REYNA, T.; REYNA, S.; LÁBAQUE, M.; FULGINITI, F.; RIHA, C.; LINARES, J. Facultad de Ciencias Exactas, Físicas y Naturales. Universidad Nacional Córdoba. Importancia de la determinación de la humedad en estudios de infiltración y escorrentía superficial para períodos largos. Ambi-Agua, Taubaté, v. 6, n. 2, p. 91-110, 2011. (doi:10.4136/ambi-agua.188)

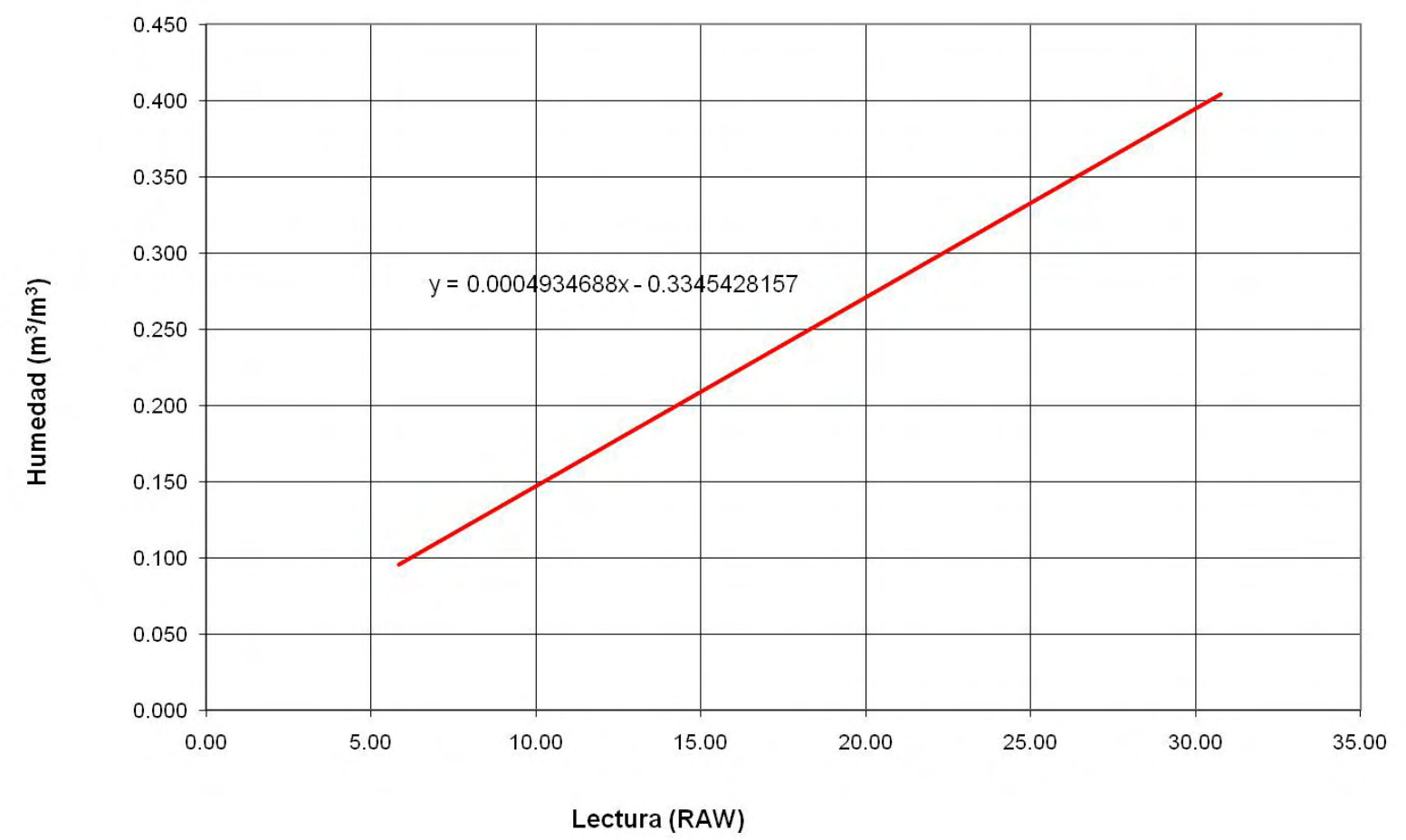

Figura 13. Función de correlación lectura (RAW) vs humedad $\left(\mathrm{m}^{3} / \mathrm{m}^{3}\right)$ para el sensor 10-HS para el suelo de la parcela del INTA.

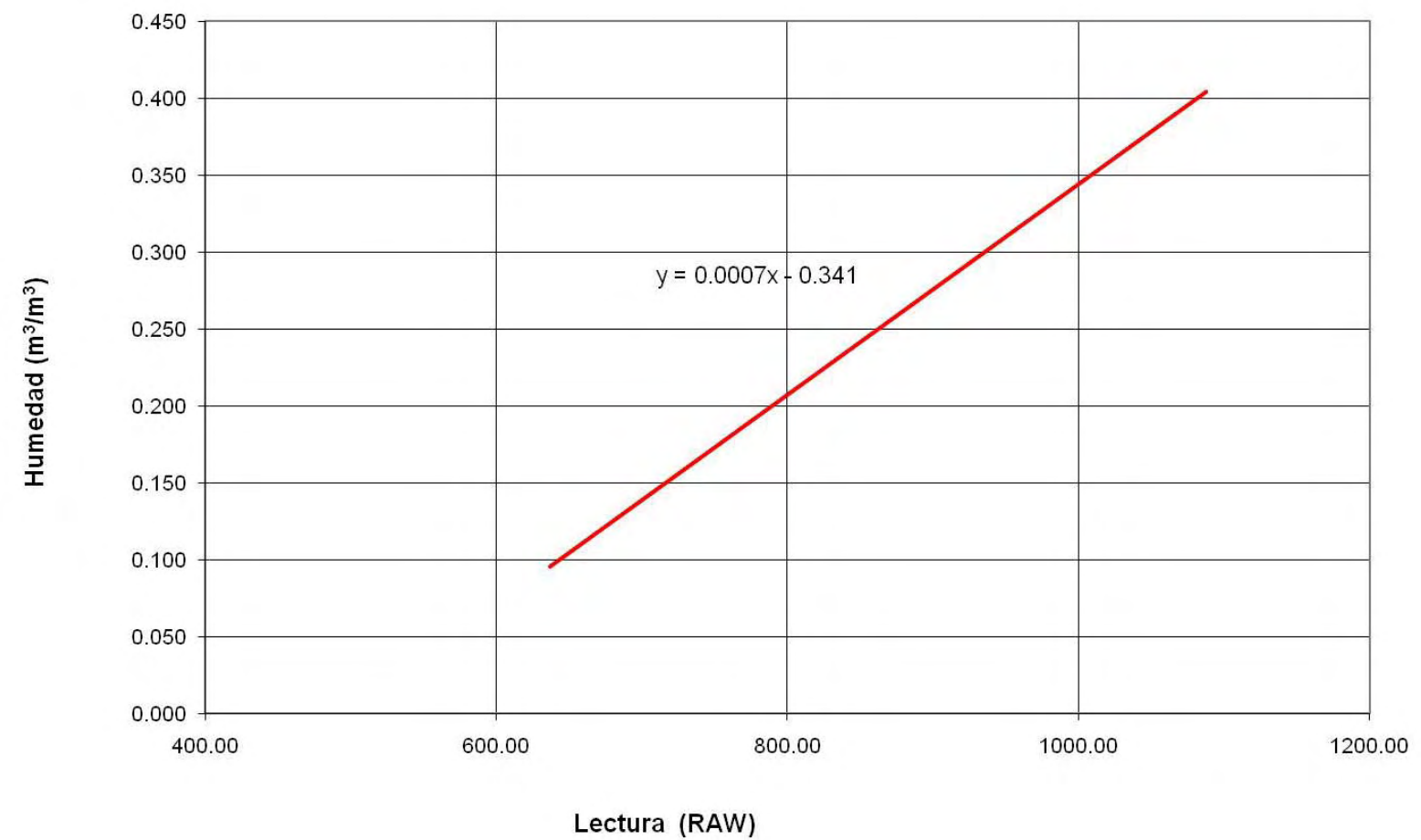

Figura 14. Función de correlación lectura (RAW) vs humedad $\left(\mathrm{m}^{3} / \mathrm{m}^{3}\right)$ para el sensor EC-5 para el suelo de la parcela del INTA.

\section{RESULTADOS}

En las Figuras 15 y 16 se presentan sólo la modelación de un evento que corresponde a la precipitación de $1 \mathrm{~mm}$ ocurrida el 17 de marzo del 2010 junto con los datos obtenidos de los sensores. Los resultados del perfil de humedad obtenidos a $10 \mathrm{~cm}$ y $30 \mathrm{~cm}$ de profundidad 
REYNA, T.; REYNA, S.; LÁBAQUE, M.; FULGINITI, F.; RIHA, C.; LINARES, J. Facultad de Ciencias Exactas, Físicas y Naturales. Universidad Nacional Córdoba. Importancia de la determinación de la humedad en estudios de infiltración y escorrentía superficial para períodos largos. Ambi-Agua, Taubaté, v. 6, n. 2, p. 91-110, 2011. (doi:10.4136/ambi-agua.188)

muestran como la humedad a los $10 \mathrm{~cm}$ es mayor que la humedad a los $30 \mathrm{~cm}$ en todo el periodo modelado presentándose una mayor variación cuando se produce la precipitación que no logra saturar la superficie.

Luego se modeló el evento ocurrido durante el día 18 de marzo de 2010 con una lámina de $3 \mathrm{~mm}$ los resultados se presentan en la Figura 17.

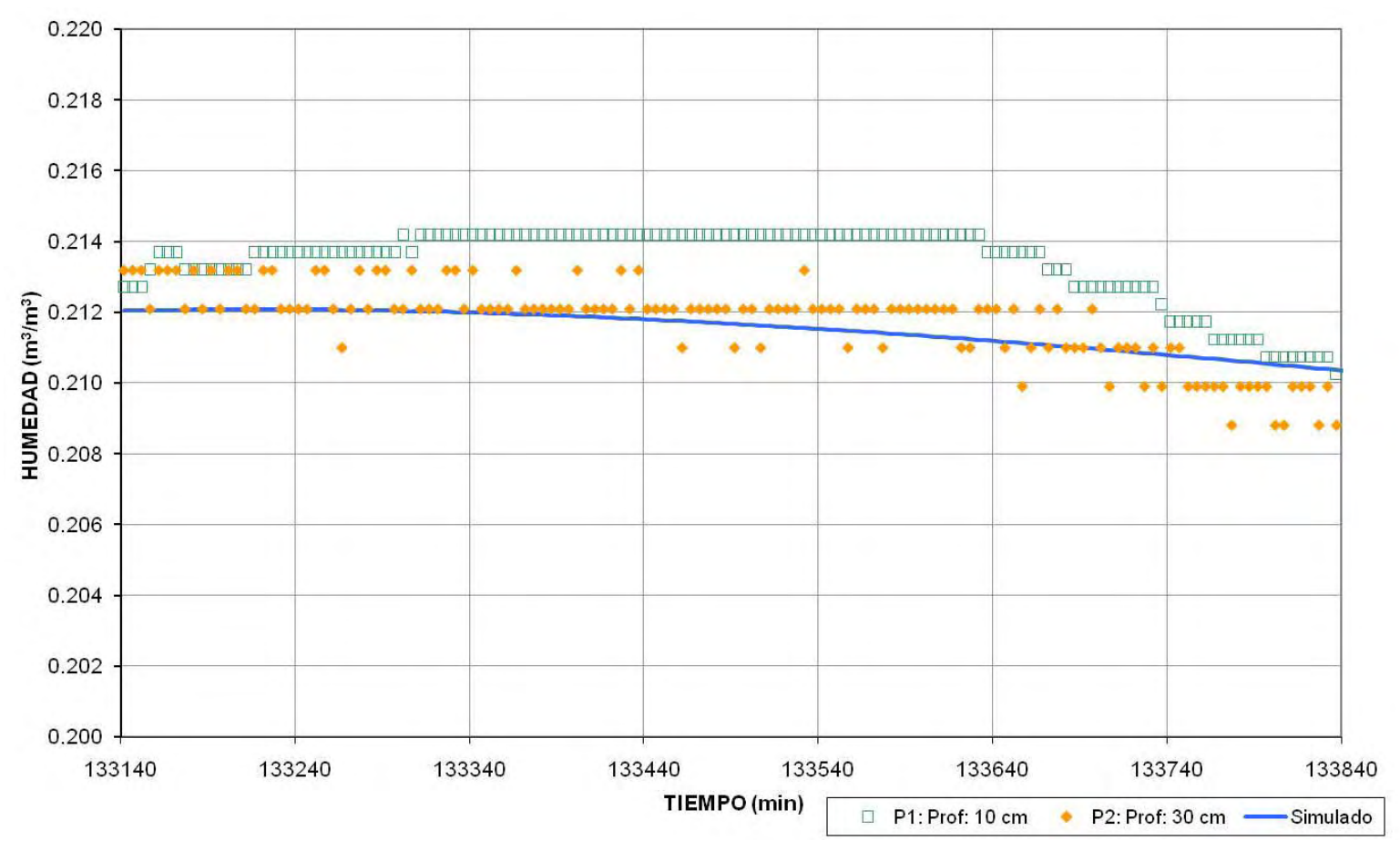

Figura 15. Curvas de humedad medidas y modelada para evento del día 07/03/2010 entre las 02:58 AM y las 02:38 PM.

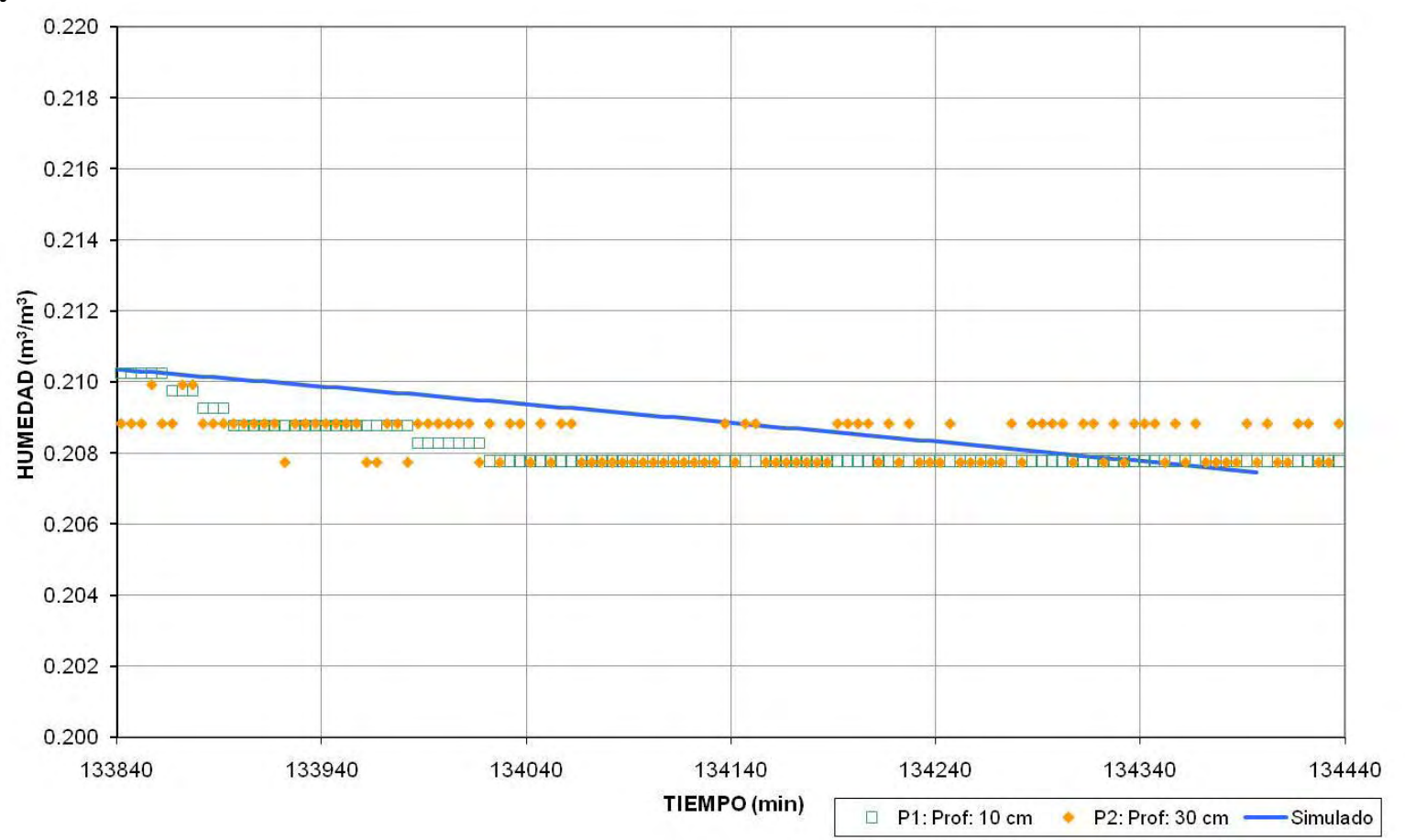

Figura 16. Curvas de humedad medidas y modelada para evento del día 07/03/2010 entre las 02:38 PM y las 00:38 AM. 
REYNA, T.; REYNA, S.; LÁBAQUE, M.; FULGINITI, F.; RIHA, C.; LINARES, J. Facultad de Ciencias Exactas, Físicas y Naturales. Universidad Nacional Córdoba. Importancia de la determinación de la humedad en estudios de infiltración y escorrentía superficial para períodos largos. Ambi-Agua, Taubaté, v. 6, n. 2, p. 91-110, 2011. (doi:10.4136/ambi-agua.188)

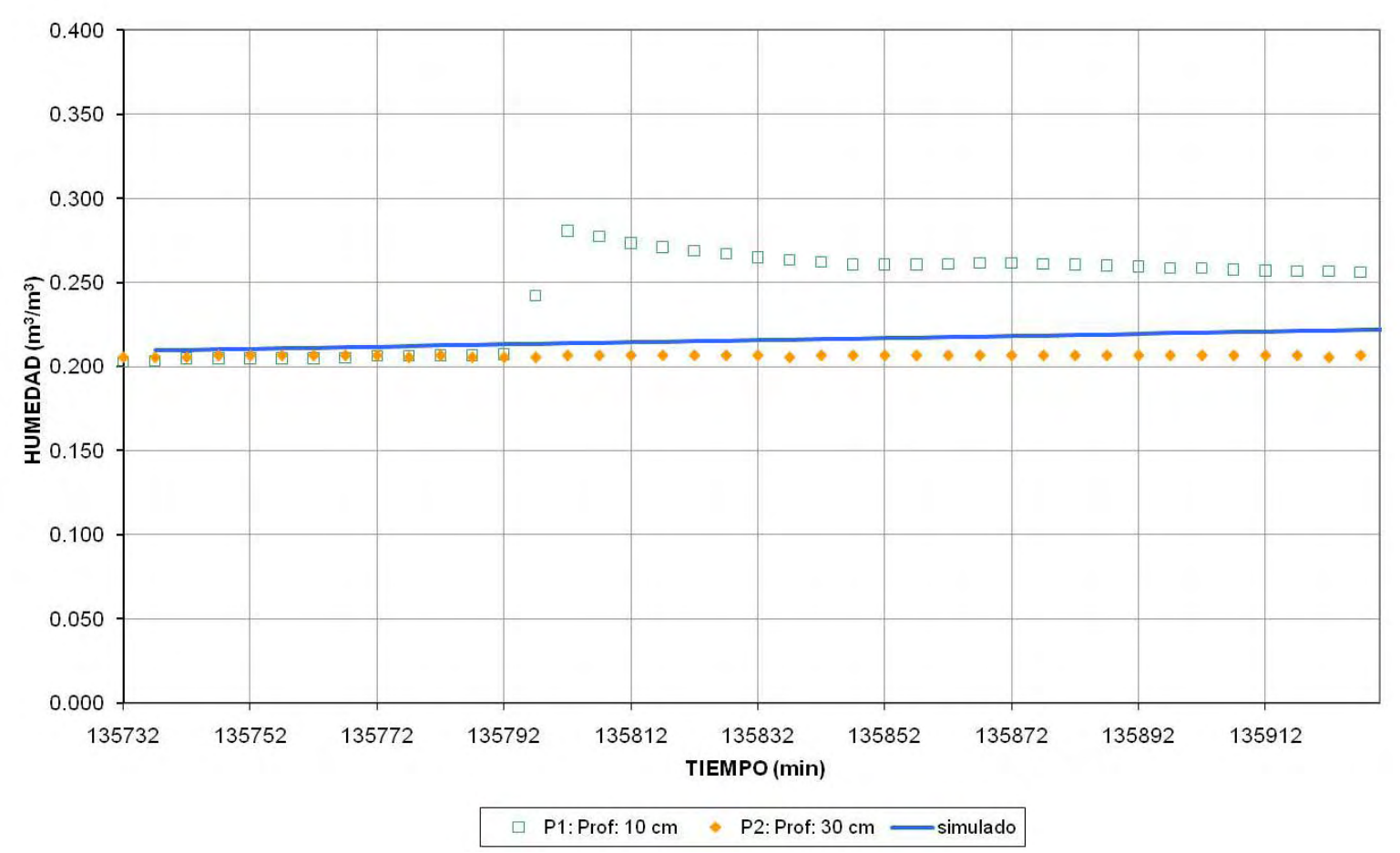

Figura 17. Curvas de humedades medidas y modelada para el evento del día 18 de marzo de 2010. (10:10 PM - 01:56AM).

Por otro lado los resultados de la modelación, muestran que las funciones hidráulicas determinadas precedentemente representan adecuadamente el comportamiento de humedecimiento y secado del suelo.

La salida de NETRAIN permite obtener el perfil de humedad para cada tiempo y la precipitación efectiva al descontar el agua que se infiltra en el suelo durante el proceso lo que caracteriza no sólo el escurrimiento superficial, sino también los estados de humedad del suelo en la zona vadosa durante y luego de estos eventos.

Este conocimiento permite determinar la capacidad disponible de humedad en el suelo para actividades agronómicas y es una herramienta importante en el estudio del transporte de contaminantes.

\section{CONCLUSIONES}

La determinación de la curva de humedecimiento superficial del suelo es primordial para resolver problemas vinculados a las necesidades de agua de riego, consideraciones ambientales y determinación de los excedentes hídricos.

De manera particular, para el cálculo del escurrimiento ante eventos continuos de lluvia, se pueden adoptar modelos simplificados (consideran a la infiltración exclusivamente como una pérdida y no dan información de las condiciones de humedad del suelo) o modelos de cálculo de infiltración (modelan el agua infiltrada y consideran más adecuadamente la interacción de los procesos de infiltración - escorrentía).

Existen en la actualidad diversos modelos numéricos que permiten predecir el movimiento del agua y los químicos en y a través de la zona no-saturada del suelo como son HYDRUS (Simunek et al., 1999); UNSATCHEM-2D (Simunek et al., 1996); CHAIN-2D (Simunek et al., 1994); MODFLOW (Harbaugh y Mc Donald, 1996); etc. Estos modelos utilizan numerosos parámetros entre ellos: las funciones hidráulicas de los suelos no- 
REYNA, T.; REYNA, S.; LÁBAQUE, M.; FULGINITI, F.; RIHA, C.; LINARES, J. Facultad de Ciencias Exactas, Físicas y Naturales. Universidad Nacional Córdoba. Importancia de la determinación de la humedad en estudios de infiltración y escorrentía superficial para períodos largos. Ambi-Agua, Taubaté, v. 6, n. 2, p. 91-110, 2011. (doi:10.4136/ambi-agua.188)

saturados. En general estas funciones se obtienen de la aplicación de modelos como los de Brooks y Corey $(1964,1966)$ y Van Genuchten (1980) entre otros.

Reyna (2000) encontró que estos modelos ajustan de manera razonable sólo en un rango de humedad cuando se analizan los suelos loéssicos de Córdoba (Argentina).

Cuando el rango de variación de la succión o la conductividad se desarrolla a través de distintos órdenes de magnitud, es necesario representar esta variación mediante funciones por tramos $\mathrm{y}$, en muchas ocasiones los parámetros requeridos por estas funciones no se encuentran disponibles. El uso y desarrollo de herramientas computacionales como NETRAIN permite subsanar estos inconvenientes.

En este artículo se describe esta herramienta computacional y el método utilizado para definir las propiedades hidráulicas a utilizar en los cálculos.

Con el fin de verificar los valores obtenidos por NETRAIN se contrastaron las curvas de humedad simuladas por este programa para un evento de lluvia, con mediciones de campo obtenidas para ese evento.

Para la obtención de las mediciones de campo y a fin de disminuir el error en la toma de datos, se realizaron, previamente, la calibración de los equipos utilizados.

Los datos de humedad registrados muestran que en los primeros $10 \mathrm{~cm}$ de suelo, el mismo acompañan a los registros de lluvia con ciclos de humedecimiento y secados. De manera más amortiguada se siguen observando estos ciclos a $30 \mathrm{~cm}$ de profundidad y pasan a ser casi imperturbados a profundidades de $80 \mathrm{~cm}$ o más.

Los resultados de contrastar los datos de la modelación con NETRAIN y los valores registrados en campo para el evento modelado a la profundidad de $30 \mathrm{~cm}$ muestran una diferencia del orden del 1\%; que es el mismo orden del error de registro de los sensores.

Actualmente se sigue tomando en campo datos de precipitación y lectura de los sensores de manera continua para luego contrastarlos con los resultados arrojados por NETRAIN modelado con los mismos eventos de precipitación y ausencia de esta.

Al mismo tiempo se han instalado nuevos sensores en parcelas de uso agrícola sometidos a riego. Estas observaciones servirán de base para estudiar el comportamiento de los plaguicidas y fertilizantes en los perfiles de los suelos y su potencial capacidad de contaminar las aguas subterráneas. Estos estudios se muestran prioritarios para poder definir pautas de manejo ambiental de los suelos sometidos a actividades agrícolas.

\section{REFERENCIAS}

BOGENA, H. R.; HUISMAN, J. A.; OBERDÖRSTER, C.; VEREECKEN, H. Evaluation of a low-cost soil water content sensor for wireless network applications. Journal Hyd., v. 344, p. 32-42, 2007. http://dx.doi.org/10.1016/j.jhydrol.2007.06.032

BREDDIN, H. Due grundribkarnten des hydrogeologischen kartenwekes der wasserwistschafteverwattung von Norgrhein-Westfalen. Geologische Mitteilungen, Aachen, v. 2, n. 4, p. 393-416, 1963.

BURKART, R., N. O. Bárbaro, R. O. Sánchez y D. A. Gómez (1999). Eco-regiones de la Argentina, Secretaría de Recursos Naturales y Desarrollo Sustentable, Buenos Aires, 42 páginas.

FRENGUELLI, J. Loess y limos pampeanos. La Plata: Univ. Nac. de la Plata, Fac. de Cs. Nat. y Museo, 1955. (Técnica y Didáctica, 7). 
REYNA, T.; REYNA, S.; LÁBAQUE, M.; FULGINITI, F.; RIHA, C.; LINARES, J. Facultad de Ciencias Exactas, Físicas y Naturales. Universidad Nacional Córdoba. Importancia de la determinación de la humedad en estudios de infiltración y escorrentía superficial para períodos largos. Ambi-Agua, Taubaté, v. 6, n. 2, p. 91-110, 2011. (doi:10.4136/ambi-agua.188)

GREEN, R. E.; COREY, J. C. Calculation of hydraulic conductivity: a further evaluation of some predictive methods. Soil. Sci. Am. Proc., v. 35, p. 3-8, 1971.

http://dx.doi.org/10.2136/sssaj1971.03615995003500010010x

HALL, A. J.; REBELLA, C. M.; GHERSA, C. M.; CULOT, J. P. Field-crop systems of the Pampas. In: PERSON, C. J. (Ed.). Field crop ecosystems: ecosystems of the world. New York: Elsevier, 1992.

HARBAUGH, A. W. Y.; MC DONALD, M. G. User`s documentation for modflow 96, an update to the U.S. geological survey modular finite: diference groundwater flow model. Reston: U. S. Geological Survey, 1996. p. 96-485. (Open File Report).

IBAÑEZ, J. J. Los suelos de Argentina y su geografía. Disponível em: $<$ http://www.madrimasd.org/>. Acesso: 2010.

JACKSON, R. D.; REGINATO, R. J.; VAN BAVEL, C. H. Comparison of measured and calculated hydraulic conductivities of unsaturated soils. Water Resour. Res., v. 1, p. 375-380, 1965. http://dx.doi.org/10.1029/WR001i003p00375

LEIJ, F. J.; ALVES, W. J.; VAN GENUCHTEN, M. T. H.; WILLIAMS, J. R. The UNSODA unsaturated soil hydraulic database: user's manual version 1.0. Cincinnati: U.S. Environmental Protection Agency; National Risk Management Research Laboratory; Office of Research and Development, 1996.

MESChtANKOVA, L. Ph.D. Thesis. Moscow: Moscow State Univ, 1989.

MUALEM, Y. A New model for predicting the hydraulic conductivity of unsaturated porous media. Water Resour. Res., v. 12, p. 513-522, 1976.

http://dx.doi.org/10.1029/WR012i003p00513

PAZ, R.; STORTI, M.; IDELSOHN, S.; RODRÍGUEZ, L.; VIONNET, C. Simulación de la interacción agua subterránea/superficial mediante un código en elementos finitos en paralelo. In: CONGRESO NACIONAL DEL AGUA, 20., 2005, Mendoza. Anales... Mendoza: [S.n.], 2005.

PAZOS, M. S.; MOSCATELLI, G. The WRB applied to pampean soils - Argentina. In: WORLD CONGRESS OF SOIL SCIENCE, 16., 1998, Montpellier. Proceedings... Montpellier: ISSS, 1998. 1 CD-ROM.

PLAGGE et al. Z. Pflanzenernaehr. Bodenkd, v. 153, p. 39-45, 1990.

REDOLFI, E. Comportamiento de pilotes en suelos colapsables. Madrid: Centro de Estudios y Experimentación de Obras Públicas, 1993.

REYNA, T. Funciones hidráulicas en suelos no saturados: aplicación al loess pampeano. 2000. Tesis (Maestría) - Facultad de Ciencias Exactas, Físicas y Naturales, Universidad Nacional de Córdoba, Córdoba, 2000.

REYNA, T. Acoplamiento de los proceso de precipitación y escorrentía. 2008. Tesis (Doctoral) - Facultad de Ciencias Exactas, Físicas y Naturales, Universidad Nacional de Córdoba, Córdoba, 2008.

RINALDI, V. Propiedades dieléctricas del loess del centro de Argentina. Tesis (Doctoral) - Facultad de Ciencias Exactas, Físicas y Naturales, Universidad Nacional de Córdoba, Córdoba, 1994. 
REYNA, T.; REYNA, S.; LÁBAQUE, M.; FULGINITI, F.; RIHA, C.; LINARES, J. Facultad de Ciencias Exactas, Físicas y Naturales. Universidad Nacional Córdoba. Importancia de la determinación de la humedad en estudios de infiltración y escorrentía superficial para períodos largos. Ambi-Agua, Taubaté, v. 6, n. 2, p. 91-110, 2011. (doi:10.4136/ambi-agua.188)

ROCKHOLD, M. L.; FAYER M. J.; GEE C. W. Characterization of unsaturated hydraulic conductivity at the hanford site., Richland: Pacific Northwest Laboratory, 1988. (Publication 6488).

SIMUNEK, J.; SUAREZ, D. L.; SEJNA, M. The UNSATCHEM software package for simulating the one - dimensional variably saturated water flow, heat transport, carbon dioxide production and transport, and multicomponent solute transport with mayor ion equilibrium and kinetic chemistry: version 2.0. Riverside: U. S. Salinity Laboratory; ARS-USDA, 1996. (Research Report, 141).

SIMUNEK, J.; Van GENUCHTEN, M. Th. The CHAIN-2D code for simulating the twodimensional movement of water, heat, and multiple solutes in variably-saturated porous media: - version 1.1. Riverside: U.S. Salinity Laboratory; ARS- USDA, 1994. (Research Report, 136).

SIMUNEK, J.; SEJNA, M.; Van GENUCHTEN, M. Th. HYDRUS-2D, simulating water flow, heat and solute transport in two - dimensional variably saturated media: version 2.0. Riverside: U.S. Salinity Laboratory; ARS- USDA, 1999.

SCOPPA, C. O. The pedogenesis of a sequence of mollisols in the Undulating Pampa (Argentina). 1974. Thesis (Doctoral Science) - State University of Ghent, Ghent, 1974.

SHEIN. Thesis. Moscow State Univ., Moscow, 1990.

TERUGGI, M. The nature and origin of Argentine loess. J. Sed. Petrol., v. 27, n. 3, p. 322332, 1957.

VAN GENUCHTEN, M. Th. A closed-form equation for predicting the hydraulic conductivity of unsaturated soils. Soil Sience Society of American Journal, v. 44, p. 892-898, 1980.

http://dx.doi.org/10.2136/sssaj1980.03615995004400050002x

VITA SERMAN, F.; SCHUGURENSKY, C.; CARRIÓN, R.; RODRÍGUEZ, S. Evaluación del comportamiento de sensores de humedad de suelo del tipo (FDR) de desarrollo local, en relación al contenido de agua y a la textura de suelo. . In: JORNADAS DE ACTUALIZACIÓN EN RIEGO Y FERTIRRIEGO, 3., 2006, Mendoza. Anales... Mendonza: INTA, 2006. 